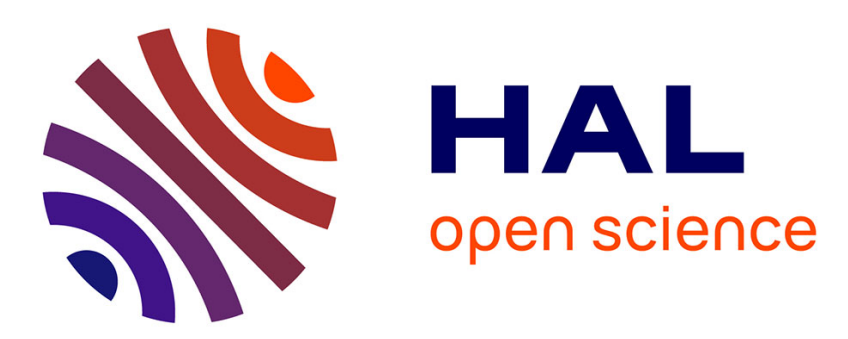

\title{
Aspects microstructuraux de la déformation cyclique dans les métaux et alliages C. C. et C.F.C. I. - Consolidation cyclique
}

\author{
T. Magnin, J. Driver, J. Lepinoux, L.P. Kubin
}

\section{- To cite this version:}

T. Magnin, J. Driver, J. Lepinoux, L.P. Kubin. Aspects microstructuraux de la déformation cyclique dans les métaux et alliages C. C. et C.F.C. I. - Consolidation cyclique. Revue de Physique Appliquée, 1984, 19 (7), pp.467-482. 10.1051/rphysap:01984001907046700 . jpa-00245219

HAL Id: jpa-00245219

https://hal.science/jpa-00245219

Submitted on 1 Jan 1984

HAL is a multi-disciplinary open access archive for the deposit and dissemination of scientific research documents, whether they are published or not. The documents may come from teaching and research institutions in France or abroad, or from public or private research centers.
L'archive ouverte pluridisciplinaire $\mathbf{H A L}$, est destinée au dépôt et à la diffusion de documents scientifiques de niveau recherche, publiés ou non, émanant des établissements d'enseignement et de recherche français ou étrangers, des laboratoires publics ou privés. 


\title{
REVUE DE PHYSIQUE APPLIQUÉE
}

\section{Aspects microstructuraux de la déformation cyclique dans les métaux et alliages C.C. et C.F.C. I. - Consolidation cyclique}

\author{
T. Magnin, J. Driver, J. Lepinoux $\left({ }^{*}\right)$ et L. P. Kubin $\left({ }^{*}\right)$ \\ E.N.S. des Mines, Département Matériaux, 158, Cours Fauriel, 42023 St Etienne Cedex, France \\ $\left({ }^{*}\right)$ Laboratoire de Métallurgie Physique $\left({ }^{+}\right)$, Faculté des Sciences, \\ 40, avenue du Recteur Pineau, 86022 Poitiers Cedex, France
}

(Reçu le ler décembre 1983, révisé le 26 mars 1984, accepté le 4 avril 1984)

\begin{abstract}
Résumé. - Cet article constitue la première partie d'une revue critique des travaux récents sur les mécanismes physiques de l'endommagement par fatigue. Les techniques modernes d'étude de la fatigue et les notions de base du comportement des dislocations dans les métaux et alliages C.C. et C.F.C. sont rappelées brièvement. La consolidation cyclique est présentée successivement sous l'aspect mécanique et sous l'aspect microstructural. L'accent est mis plus particulièrement sur l'influence de mécanismes fondamentaux tels que : friction de réseau, glissement dévié... dans les monocristaux de métaux ou d'alliages de haute pureté.
\end{abstract}

\begin{abstract}
This article is the first part of a critical review of recent work on the physical mechanisms of fatigue damage. Both modern techniques for fatigue studies and the basic notions of dislocation behaviour in F.C.C. and B.C.C. metals and alloys are briefly reviewed. Cyclic hardening is described from both mechanical and microstructural point of view. Particular emphasis is placed on the influence of the fundamental mechanisms such as lattice friction, stacking fault energy... in single crystals of high purity metals and alloys.
\end{abstract}

\section{Introduction.}

Il est reconnu depuis déjà longtemps que la rupture des métaux et alliages dans des conditions de sollicitation cyclique intervient comme conséquence de la localisation de la déformation plastique au cours de la fatigue. Afin de comprendre et de prévoir l'endommagement (et la durée de vie) d'un métal ou d'un alliage métallique dans ces conditions, il apparaît indispensable de connaître les mécanismes microstructuraux de la déformation plastique cyclique. Une telle démarche ne peut que bénéficier des résultats obtenus lors d'études de plasticité en déformation unidirectionnelle car si les conditions de sollicitation sont alors

$\left(^{+}\right)$L.A. 131 CNRS différentes les propriétés fondamentales des dislocations restent nécessairement les mêmes.

Dans les deux cas, on peut donc espérer réaliser un lien entre l'évolution de la microstructure et les propriétés mécaniques macroscopiques, et non plus se contenter d'établir des relations phénoménologiques dont on ne peut garantir la capacité de prédiction.

De nombreuses études ont été menées dans ce sens depuis une quinzaine d'années et l'on trouvera par ailleurs d'excellentes revues (en anglais) concernant essentiellement le comportement cyclique de quelques métaux cubiques à faces centrées purs [1-3]. Plus récemment, des progrès importants ont été réalisés dans la compréhension des mécanismes de l'endommagement par fatigue et dans l'élaboration de modèles « physiques » semi-quantitatifs. Ces progrès sont essen- 
tiellement dus à la mise en œuvre de techniques d'essais et d'observations mieux adaptées à l'étude de la plasticité microstructurale : essais de fatigue à amplitude et vitesse de déformation plastique imposées, souvent réalisés sur monocristaux, et se prềtant ainsi à l'observation par les différentes techniques de la microscopie optique et électronique. L'objectif de la présente revue consiste à donner une vue critique des travaux récents reliant ainsi l'aspect microstructural et l'aspect mécanique de l'endommagement par fatigue, jusqu'au stade de l'amorçage des fissures (les stades ultérieurs relevant plutôt de la mécanique de la rupture). Nous nous limiterons au domaine de la fatigue à froid ( $T<0,3 T_{\mathrm{F}}, T_{\mathrm{F}}$ température de fusion, c'est-à-dire en excluant tout mécanisme faisant intervenir la diffusion à longue distance) et au cas des matériaux simples les plus étudiés, tels que les métaux cubiques centrés (C.C.) ou cubiques à faces centrées (C.F.C.) purs, monophasés ou contenant des précipités, sous forme de mono- ou polycristaux. Il s'agit de dégager les progrès essentiels réalisés et les points encore au stade de la discussion, et d'illustrer leur intérêt pour une meilleure compréhension et une meilleure prévision de la tenue en fatigue.

Dans cette première partie (I) sont rappelées brièvement les techniques actuelles d'étude de la fatigue et les notions de base du comportement des dislocations, puis les mécanismes de consolidation cyclique (sur monocristaux). Les matériaux C.C. et C.F.C. sont présentés en parallèle, afin de préciser plus clairement l'influence des propriétés particulières des dislocations dans chacune de ces structures.

La seconde partie (II) traite des mécanismes de saturation cyclique des monocristaux C.C. et C.F.C. et fait le point sur les connaissances actuelles concernant les causes microscopiques de l'endommagement en surface. Enfin, un effort particulier est consacré à réunir les données permettant d'établir une corrélation entre le comportement des monocristaux et celui des polycristaux.

Dans ce qui suit, nous utilisons les notations usuelles : $\tau$ pour les contraintes de cisaillement (cisions), $\gamma$ pour le cisaillement cristallographique, $\sigma$ et $\varepsilon$ pour les contraintes et déformations axiales. $\varepsilon_{\mathfrak{t}}, \varepsilon_{\mathrm{p}}$ représentent respectivement la déformation totale (élastique plus plastique), et la déformation plastique. La notation $\Delta$ devant ces quantités (i.e. $\Delta \varepsilon_{\mathrm{p}}, \Delta \sigma$ ) est parfois utilisée pour représenter la variation maximale enregistrée au cours d'un cycle. L'indice s est réservé aux valeurs des différents paramètres lors de la saturation cyclique, et les indices $\mathbf{v}$ et $\mathrm{c}$ aux quantités se rapportant aux dislocations vis et coin respectivement. Nous utiliserons les abréviations BGP pour bandes de glissement persistantes (PSB en anglais), et EDE pour l'énergie de défaut d'empilement (ou énergie de faute) qui sera notée $\Gamma$ (et non $\gamma$ ) afin d'éviter toute confusion. Enfin, les concentrations en éléments d'alliage seront données en pourcentages atomiques (ainsi $\mathrm{Cu}-7 \mathrm{Al}$ signifie $\mathrm{Cu}-7 \%$ at. $\mathrm{Al}$ ).

\section{Notions de base et méthodes d'analyse.}

2.1 L'ENDOMMAGEMENT PAR fatigue : PARAmÈtres DE BASE ET MÉTHODES D'ESSAI. - L'endommagement des matériaux métalliques sollicités en fatigue résulte essentiellement de leur déformation plastique cyclique. Cette déformation peut être soit homogène, soit hétérogène à l'échelle macroscopique (congés de raccordement, fonds de fissures) ou à l'échelle microscopique (joints de grains...). Ce sont l'amplitude et la répartition des déformations plastiques qui régissent l'écrouissage cyclique, l'amorçage et, à un degré moindre, la propagation des fissures de fatigue. Bauschinger [4] fut le premier à reconnaître l'importance de la déformation plastique sur les caractéristiques mécaniques en fatigue; ce concept fut confirmé par la suite par de nombreuses études désormais classiques (Ewing et Humphrey [5], Gough [6], Cazaud [7], Coffin [8]).

Les amplitudes des déformations plastiques vont de valeurs très faibles $\left(\leqslant 10^{-6}\right)$ à des valeurs très importantes (par exemple $\sim 1$ en fond de fissure). Elles sont habituellement déterminées par des essais de cyclage (généralement en traction-compression) sur des éprouvettes lisses, avec mesure simultanée des efforts et des déplacements. A chaque cycle d'effort, si la déformation n'est pas parfaitement élastique, une boucle d'hystérésis est constatée sur la courbe contrainte-déformation (Fig. 1). Les durées de vie $\left(N_{\mathrm{R}}\right)$ en fatigue plastique sont souvent reliées directement à $\Delta \varepsilon_{\mathrm{p}}$ par la loi dite de Manson-Coffin :

$$
\Delta \varepsilon_{\mathrm{p}} \times N_{\mathrm{R}}^{\alpha}=C
$$

où $\alpha$ et $C$ sont des constantes.

Pour analyser le comportement des matériaux en fatigue, trois types d'essais sont couramment employés : 1) Cyclage à contrainte et fréquence constantes. 2) Cyclage à amplitude et vitesse de déformation totale constantes. 3) Cyclage à amplitude et vitesse de déformation plastique constantes. Ces techniques d'essais sont décrites en détail dans de nombreux ouvrages $[9,10]$. Le type d'essai peut influer sur les résultats obtenus, essentiellement à cause des effets de durcissement ou d'adoucissement cycliques (à déformation imposée, respectivement augmentation ou diminution de la contrainte d'écoulement maximale). Lors des essais à $\Delta \varepsilon_{\mathrm{t}}$ ou $\Delta \sigma$ constants, il y a souvent une évolution de la déformation plastique à chaque cycle. A cause de cet effet qui est surtout sensible à $\Delta \varepsilon_{\mathrm{p}} / 2 \leqslant 10^{-3}$, il est beaucoup plus satisfaisant de solliciter le matériau à $\Delta \varepsilon_{\mathrm{p}}$ constant et d'enregistrer l'évolution de $\Delta \sigma$. De plus, ce type d'essai fournit les valeurs exactes de la déformation plastique cumulée $\left(\varepsilon_{\text {p cum }}=2 . \Delta \varepsilon_{\mathrm{p}} \cdot N, N\right.$ étant le nombre de cycles) et permet une meilleure corrélation entre les propriétés mécaniques cycliques et les microstructures. Sur la figure 1 qui montre l'évolution des boucles d'hystérésis pour un polycristal d'Al-Mg à $\Delta \varepsilon_{\mathrm{p}} / 2$ 


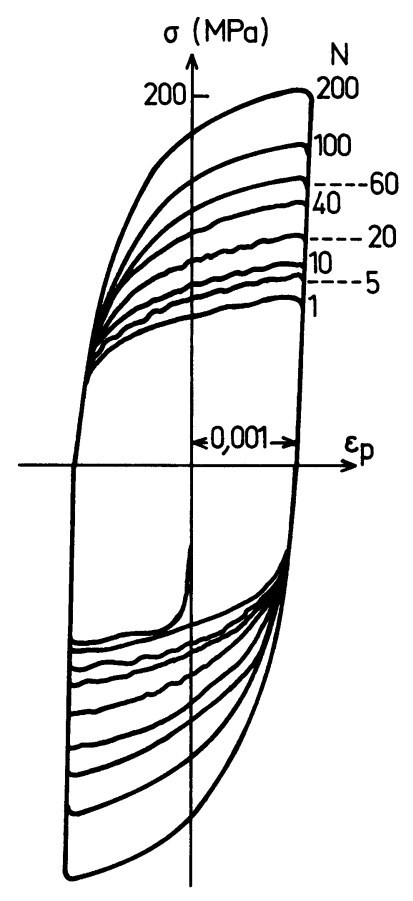

$\mathrm{Al}-5 \mathrm{Mg}, \frac{\Delta \varepsilon_{\mathrm{p}}}{2}= \pm 10^{-3}$

Fig. 1. - Evolution des boucles d'hystérésis en fonction du nombre de cycles d'un alliage $\mathrm{Al}-5 \mathrm{Mg}$ à $\Delta \varepsilon_{\mathrm{p}}$ imposé (J. Driver, travaux non publiés).

[Hysteresis loops of an Al-5 Mg alloy at constant $\Delta \varepsilon_{\mathrm{p}}$ as a function of the number of cycles (J. Driver, unpublished work).]

imposé, un dụrcissement important est observé au début du cyclage. Cependant, après un certain nombre de cycles la contrainte n'évolue plus : l'état de saturation est atteint. La contrainte correspondante $\left(\Delta \sigma_{\mathrm{s}} / 2\right)$ est fonction de l'amplitude de la déformation plastique imposée, et les courbes $\Delta \sigma_{\mathrm{s}} / 2=f\left(\Delta \varepsilon_{\mathrm{p}} / 2\right)$ sont appelées " courbes d'écrouissage cyclique ", et souvent comparées aux courbes de déformation monotone (cf. Fig. 2).

\section{2 OBSERVATION DES ASPECTS MICROSTRUCTURAUX.}

2.2.1 Aspects généraux du comportement des dislocations dans les réseaux C.F.C. et C.C. - Cas des monocristaux C.F.C. - L'énergie de défaut d'empilement $(\Gamma)$ détermine dans ce cas les caractéristiques essentielles de la microstructure. Une faible énergie de faute $\left(\Gamma \sim\right.$ quelques $\left.\mathrm{mJ} / \mathrm{m}^{2}\right)$ favorise le mouvement de dislocations partielles et inhibe donc le glissement dévié ; la déformation a alors tendance à devenir planaire et hétérogène. Une forte énergie de faute, en revanche, favorise le glissement dévié et

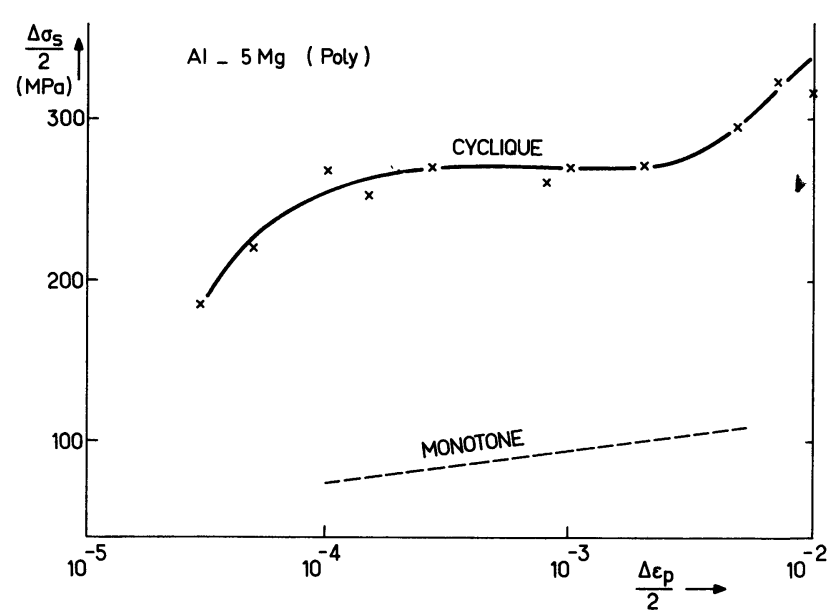

Fig. 2. - Courbes d'écrouissage cyclique et monotone d'un alliage Al-5 $\mathrm{Mg}$ polycristallin [43].

[Cyclic and monotonic hardening curves of a polycrystalline Al-5 Mg alloy [43].]

attribue un degré de liberté supplémentaire aux dislocations vis, rendant ainsi la microstructure tridimensionnelle et plus homogène. On trouvera des valeurs tabulées de $\Gamma$ pour les métaux C.F.C. purs dans $[11,12]$. Elles vont de $20 \mathrm{~mJ} / \mathrm{m}^{2}$ pour l'argent à environ $150 \mathrm{~mJ} / \mathrm{m}^{2}$ pour l'aluminium. Notons toutefois qu'en règle générale il est plus correct pour comparer deux matériaux d'utiliser la quantité sans dimensions $\Gamma / \mu b$, où $\mu$ est le module de cisaillement et $b$ le module du vecteur de Burgers.

L'évolution des microstructures en déformation uniaxiale monotone, à moyenne et basse température et en fonction du paramètre $\Gamma / \mu b$ a fait l'objet de nombreuses revues [13-16]. En l'absence de friction de réseau, ce sont les interactions entre dislocations mobiles et dislocations fixes qui déterminent la limite élastique; elles conduisent à une déformation en trois stades. Le stade I est caractérisé par la formation de longs dipôles, résultant de l'interaction entre dislocations mobiles sur des plans voisins. Dans le stade II, il y a interférence entre le système de glissement primaire et les systèmes secondaires. Lorsque $\Gamma / \mu b$ est élevé, les réarrangements locaux par glissement dévié conduisent à la formation d'une structure cellulaire, dont l'illustration typique est donnée figure $3 \mathrm{a}$ par le cas de l'aluminium. Lorsque $\Gamma / \mu b$ décroît, on observe, dans l'ordre les effets suivants : décroissance de la taille des cellules, les sous-joints devenant moins réguliers, puis formation d'empilements de dislocations (Fig. 3b) et possibilité de formation de jonctions entre dislocations partielles (barrières de LomerCottrell). Aux très faibles valeurs de $\Gamma / \mu b$, on aboutit à une interaction entre systèmes de glissement planaires. La possibilité de mouvement de dislocations partielles favorise en outre la déformation par maclage. 


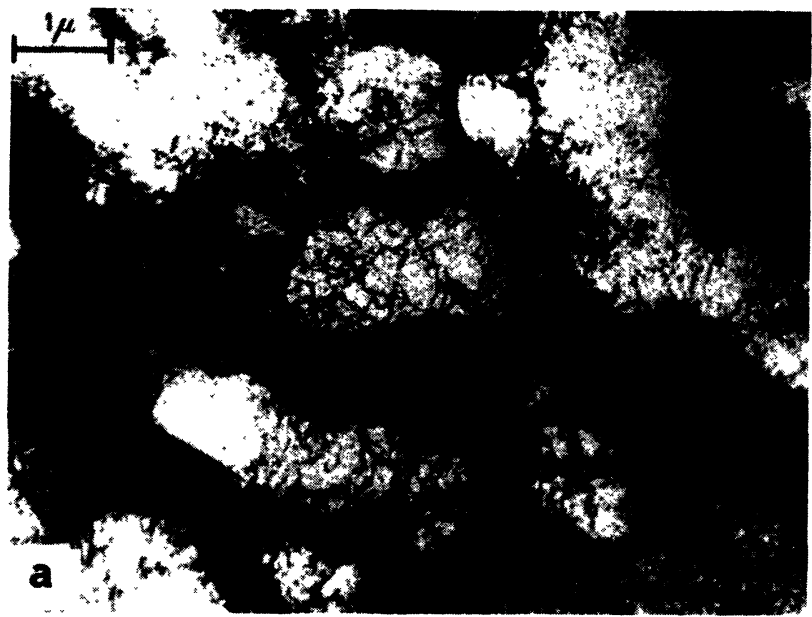

Fig. 3a. - Structure cellulaire observée dans l'aluminium après une déformation en traction de $10 \%$ à $77 \mathrm{~K}$ [15].

[Dislocation cell structure in aluminium deformed $10 \%$ in tension at $77 \mathrm{~K}$ [15].]

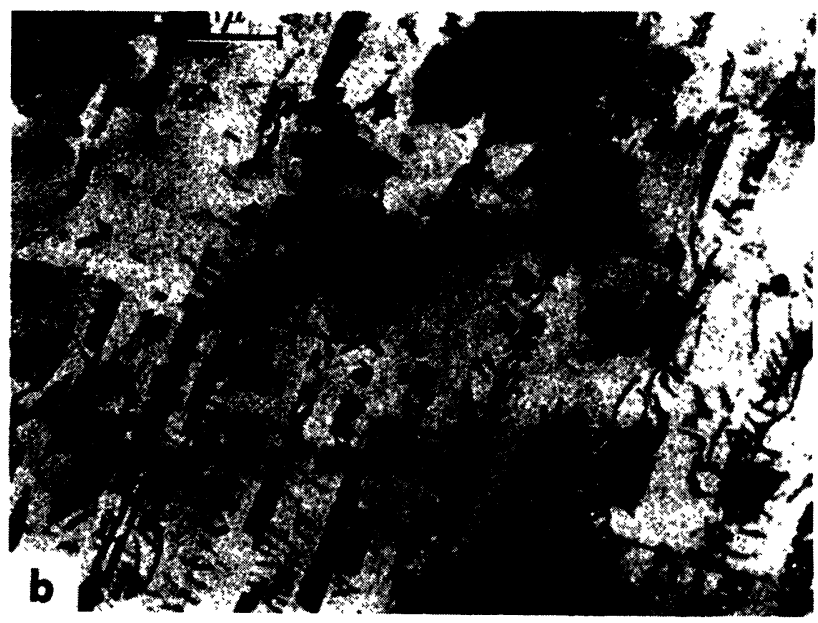

Fig. 3b. - Formation d'empilements de dislocations dans un alliage $\mathrm{Cu}-8 \% \mathrm{Al}$ après une déformation de $5 \%$ en traction [15].

[Dislocation pile-ups in a $\mathrm{Cu}-8 \% \mathrm{Al}$ alloy after $5 \%$ tensile deformation [15].]

Enfin, le stade III est caractérisé par la relaxation des contraintes internes au moyen du glissement dévié. La possibilité d'opération de ce dernier mécanisme est cependant étroitement liée aux valeurs de la température, et de l'EDE [17].

Cas des monocristaux C.C. - Contrairement à ce qui se passe dans les métaux C.F.C., la contrainte d'écoulement des C.C. dépend fortement de la température et de la vitesse de déformation lorsque
$T<0,2 T_{\mathrm{f}}$ [18-21]. Ce fait est lié au rôle particulier des dislocations vis $a / 2\langle 111\rangle$ dans le réseau C.C. Le phénomène essentiel est constitué par la structure de cour de ces dislocations que l'on peut considérer comme faiblement dissociées (quelques $b$ ) sur plusieurs plans $\{110\}$ ou $\{112\}$ en zone. La faible mobilité de cette structure tridimensionnelle est à l'origine d'une forte friction de réseau (ou contrainte de Peierls) qui, par le biais de l'activation thermique, se trouve dépendre fortement de la température (typiquement $10^{-2} \mu$ au zéro absolu, et $5 \times 10^{-4} \mu$ aux températures moyennes). Par contraste, les dislocations coins et mixtes sont mobiles à faible contrainte, comme dans les métaux C.F.C., et leur mobilité dépend peu de la température (à contrainte donnée). A partir de ces considérations, la contrainte appliquée, $\sigma$, peut se décomposer en deux termes : un terme "athermique " $\sigma_{\mathrm{i}}$, appelée " contrainte interne » et correspondant à l'interaction élastique à longue distance entre dislocations, et un terme thermique $\sigma^{*}$, appelé " contrainte effective ", sensible aux variations de la vitesse de déformation et de la température, et qui rend compte des obstacles à courte distance, c'est-à-dire essentiellement la friction de réseau sur les vis. En conséquence, les métaux et alliages C.C. présentent un comportement essentiellement dicté par le rapport des mobilités des dislocations coins (ou mixtes) et vis : $V_{\mathrm{c}} / V_{\mathrm{v}}$. On distingue schématiquement deux régimes :

- Pour $V_{c} \gg V_{v}$, la microstructure est dictée par la faible mobilité des dislocations vis et le haut niveau de contrainte requis pour accommoder la vitesse de déformation imposée $\left(\sigma^{*}>\sigma_{\mathrm{i}}\right)$. C'est le cas pour les métaux C.C. en dessous d'une température dite athermique, $T_{\mathrm{a}}\left(T_{\mathrm{a}} \sim 300 \mathrm{~K}\right.$ pour $\mathrm{Nb}$ et $\mathrm{Fe}, T_{\mathrm{a}} \sim 550 \mathrm{~K}$ pour Mo). On observe alors des boucles de dislocations très allongées dans la direction vis, car les dislocations coins sont beaucoup plus mobiles. Le rapport des densités $\rho_{\mathrm{v}} / \rho_{\mathrm{c}}$ est alors très supérieur à 1 . L'interaction avec le réseau prime sur'les interactions des dislocations vis avec la forêt, d'où une microstructure de dislocations rectilignes suivant les directions $\langle 111\rangle$ (cf. Fig. 4), et répartie de manière homogène dans les plans de glissement, même à forte déformation. La géométrie du glissement est complexe (plans $\{110\}$, $\{112\},\{123\}$ ou $\{h k l\}$ en zone autour de $\langle 111\rangle)$. De plus un changement du sens de la contrainte appliquée peut changer le système de glissement actif. Seule une considération détaillée de la structure de coeur des dislocations vis permet de rendre compte qualitativement de ces particularités [22].

- Pour $V_{c} \sim V_{v}$, on retrouve un comportement plus semblable à celui des C.F.C., avec boucles de dislocations isotropes et une densité de coins comparable à celle des vis. Il existe deux manières d'obtenir une telle situation : 1) A basse température $\left(T \ll T_{\mathrm{a}}\right)$, une forte concentration en éléments d'alliage produit dans les solutions solides une réduction substantielle de $V_{c}$ en raison des interactions dislocations coins- 


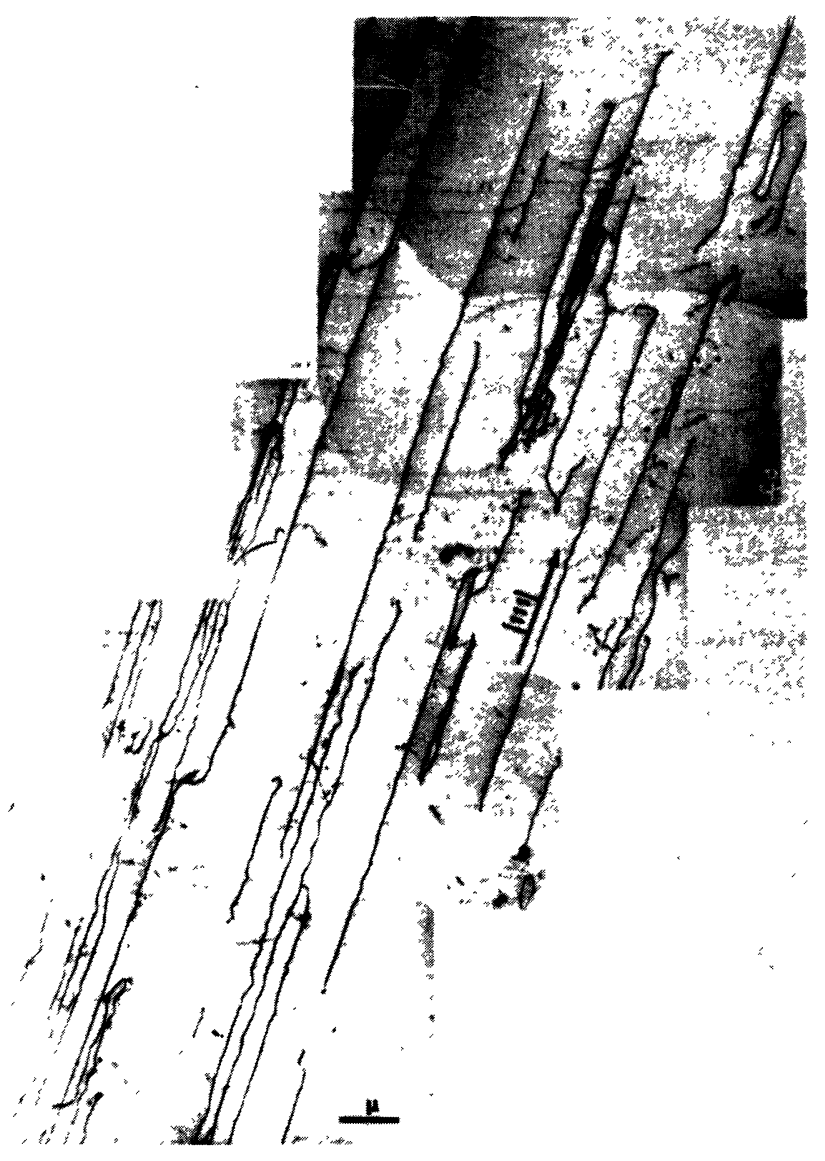

Fig. 4. - Dislocations rigides dans le $\mathrm{Nb}$ déformé en traction à $50 \mathrm{~K}$ [21a].

[Rigid dislocations in $\mathrm{Nb}$ after tensile deformation at $50 \mathrm{~K}$ [21a].]

atomes de soluté. La formation de la microstructure reste contrôlée par des interactions locales à forte contrainte critique (forces de friction, atomes de soluté) plutôt que par les interactions dislocationdislocation. La microstructure reste donc homogène tandis que le rapport $\rho_{\mathrm{c}} / \rho_{\mathrm{v}}$ tend vers 1 .

2. Aux températures moyennes $\left(T>T_{\mathrm{a}}\right)$, l'activation thermique efface progressivement les forces de friction de réseau, de sorte que l'on tend vers une situation où $\sigma_{i}>\sigma^{*}$. Les interactions entre dislocations qui déterminent $\sigma_{\mathrm{i}}$ deviennent prédominantes, et le comportement tend alors à ressembler à celui d'un métal C.F.C. à forte EDE.

Un cas intéressant est celui du fer $\alpha$, pour lequel la transition entre les deux domaines $\left(T \sim T_{\mathrm{a}}\right)$ se situe, pour les vitesses de déformation courantes $\left(\sim 10^{-4} / \mathrm{s}\right)$, au voisinage de la température ambiante. Compte tenu de la nature activée des forces de friction, une forte vitesse $\left(10^{-2} / \mathrm{s}\right)$ favorisera la formation d'une microstructure du type "basse température", avec de longs segments vis rectilignes, tandis qu'une faible vitesse $\left(10^{-5} / \mathrm{s}\right)$ conduira à la formation de multipôles coins ou de cellules suivant le taux de déformation.

2.2.2 Méthodes mécaniques d'approche des états microstructuraux. - Il est possible d'obtenir à partir d'essais mécaniques simples des renseignements sur les mécanismes microstructuraux des matériaux testés. C'est le but des méthodes mécaniques de relaxation de la contrainte, de changement de vitesse de déformation et de la méthode dite du " dip test » [23], qui permettent, au cours d'un essai de déformation monotone ou cyclique, d'avoir accès aux valeurs de $\sigma^{*}$ et $\sigma_{\mathrm{i}}$ ainsi qu'au volume d'activation $V^{*}$ (schématiquement, le volume élémentaire dans lequel s'effectue le franchissement thermiquement activé d'un obstacle). Ainsi de fortes valeurs de $\sigma^{*}$ liées à de faibles valeurs de $V^{*}\left(\sim 10 b^{3}\right)$ indiquent un régime de déformation largement marqué par de fortes contraintes de friction, alors qu'une prépondérance de $\sigma_{\mathrm{i}}$ et des valeurs élevées de $V^{*}$ $\left(\sim 1000 b^{3}\right)$ caractérisent des interactions du type forêt par exemple. On trouvera par ailleurs le principe de ces méthodes, une revue critique et détaillée de leurs applications et de leurs domaines de validité ainsi que les techniques expérimentales correspondantes [23-26].

\subsubsection{Méthodes de microscopie optique et électronique.} - Les observations microstructurales en fatigue utilisent les méthodes usuelles de la métallographie pour l'analyse des faciès d'échantillons déformés, et de la microscopie électronique pour l'étude des arrangements de dislocations. Les références données ci-après correspondent à des travaux où une méthode expérimentale particulière a été utilisée sur des échantillons cycliquement déformés.

Observations en surface. L'identification des traces et bandes de glissement, pliages en genoux... se fait notamment par l'utilisation de techniques optiques telles que la microscopie interférentielle [27], le polissage répété d'un échantillon entre deux tests et l'attaque chimique [28]. La microscopie électronique à balayage $(\mathrm{MEB})$ en réflexion allie une excellente profondeur de champ à une bonne résolution, jusqu'à $\sim 10 \mathrm{~nm}[29,30]$ et se prête bien à toutes les observations de reliefs de surface et de fissuration, permettant ainsi une bonne observation des intrusions et extrusions [31, 32]. Pour l'observation d'un relief en creux assez profond (intrusions), on peut utiliser les techniques de répliques ou observer des coupes transverses de l'échantillon (coupes par un plan contenant son axe) [32], cf. figure 5. Enfin, la prise de vues stéréographiques s'avère très aisée avec le MEB.

Etude des structures de dislocations. Les observations classiques en microscopie électronique à transmission (MET) ont pour but de déterminer le caractère et la nature des dislocations présentes dans la sous-structure (voir par exemple [33] pour une monographie), ainsi que leur arrangement qui est le plus souvent 


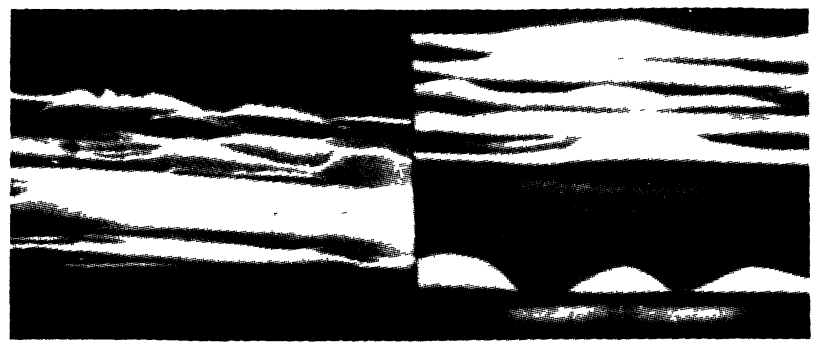

Fig. 5a. - Observation en M.E.B. d'une réplique de la surface d'échantillons de $\mathrm{Cu}$ après fatigue [32].

[S.E.M. micrograph of a surface replica taken from a fatigued Cu specimen [32].]

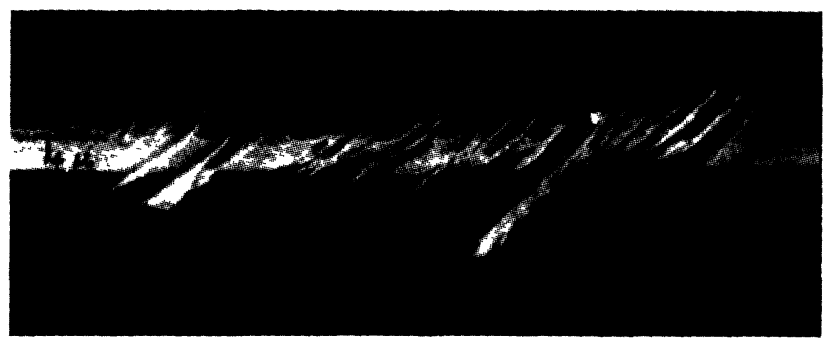

Fig. 5b. - Détail d'une bande de glissement observée dans le $\mathrm{Cu}$ après fatigue (M.E.B.) [32].

[Detail of a slip band in fatigued Cu (S.E.M.) [32].]

tridimensionnel avec des distances caractéristiquęs de l'ordre $\mathrm{du} \mu \mathrm{m}$. Une difficulté souvent rencontrée résulte de la forte densité de dislocations dans certaines configurations $\left(\sim 10^{15} / \mathrm{m}^{2}\right)$. Dans une lame trop mince (quelque $10 \mathrm{~nm}$ ), on s'attend à des pertes substantielles de dislocations lors de l'amincissement, tandis qu'en lame épaisse (1 à quelques $\mu \mathrm{m}$ en microscopie à haute tension), le recouvrement des contrastes individuels de dislocations ne permet de distinguer aucun détail. La reconstruction tridimensionnelle des structures s'effectue à partir de l'observation de lames minces taillées suivant différents plans cristallographiques [34]. La méthode des faisceaux cinématiques faibles (weak-beam) permet, en réduisant la largeur des images de dislocations, d'obtenir une bonne résolution et de mieux visualiser les détails des structures denses [35].

Le problème de la modification des sous-structures lors de la préparation des lames minces peut être crucial, mais dépend étroitement de la nature du matériau. Si dans les enchevêtrements de dislocations rencontrés en fatigue les dislocations coins et mixtes sont relativement bien ancrées par leurs interactions, les dislocations vis présentes dans les zones moins denses peuvent s'éliminer par glissement dévié à la surface de la lame. Ceci s'applique surtout aux métaux C.F.C. purs, et moins aux C.C. où les forces de friction et/ou une faible concentration d'interstitiels suffisent à immobiliser les dislocations vis. La technique d'ancrage de la sous-structure par irradiation aux neutrons sous charge (à la fin de la déformation cyclique) a été principalement développée en Allemagne [36], et s'est avérée particulièrement fructueuse dans le cas du cuivre, et dans celui du fer pur à $T>T_{\mathrm{a}}$. Les configurations de dislocations restent figées sous contrainte car elles sont ancrées par les défauts d'irradiation; on peut alors mesurer, à partir des courbures, les contraintes locales ressenties par les dislocations.

Enfin, les méthodes de déformation in situ [37] ont été appliquées à l'étude de la fatigue [38-42]. La déformation cyclique est alors effectuée sous le faisceau du microscope électronique sur une lame mince, en général pré-fatiguée, de manière à observer le comportement dynamique des dislocations sous contrainte alternée (cisaillement alterné, traction-compression ou flexion alternée).

\section{Mécanismes de consolidation cyclique.}

Nous considérons successivement les métaux et alliages C.F.C. et C.C. Dans chaque cas, nous décrivons d'abord les différents aspects du comportement mécanique cyclique, puis les évolutions correspondantes de la microstructure.

\subsection{Consolidation CyCliQue DES MÉtauX ET ALLIAGES C.F.C. MONOPHASÉS.}

3.1.1 Comportement mécanique. - L'étude de la consolidation cyclique revient à examiner l'évolution de la contrainte maximale atteinte en fin de chaque demicycle en fonction du nombre de cycles et de l'amplitude de déformation. Il est souvent possible de distinguer deux stades de consolidation : un stade initial d'évolution rapide de la contrainte (durcissement pour les métaux recuits) et un stade de saturation où la contrainte reste constante ou quasi-constante. L'étude de la forme des boucles d'hystérésis permet parfois une caractérisation plus fine du comportement mécanique.

Le durcissement cyclique. Les figures 6 et 7 représentent respectivement les courbes de consolidation cyclique de monocristaux de cuivre et de l'alliage $\mathrm{Cu}-7 \mathrm{Al}$ pour différentes amplitudes de déformation. Le durcissement initial de ces matériaux est d'autant plus prononcé que la déformation et l'EDE sont élevées [44-47]. Dans les métaux purs et les alliages peu dilués la contrainte se stabilise, bien avant rupture, après des déformations plastiques cumulées $\varepsilon_{\mathrm{p}, \text { cum }}$ de l'ordre de 5 à 10 . Dans les alliages $\mathrm{Cu}-\mathrm{Al}$ fortement alliés $(11 \%$ at.) ou même moyennement alliés (par exemple $7 \%$ at.) à $\Delta \varepsilon_{\mathrm{p}}$ faible la saturation n'est jamais vraiment atteinte, le cristal durcit lentement pour des $\varepsilon_{\mathrm{p}, \text { cum }}>100$, jusqu'à rupture.

Le stade de saturation. Pour le stade de saturation, caractéristique des métaux purs, la valeur de la contrainte de saturation, $\sigma_{\mathrm{s}}$, dépend de l'amplitude de la déformation, de la température et éventuellement, 


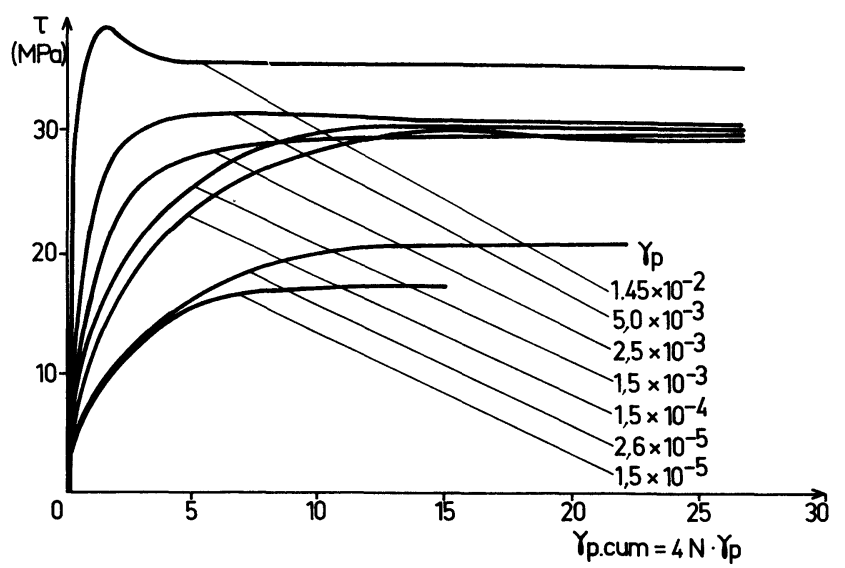

Fig. 6. - Courbes de consolidation cyclique du $\mathrm{Cu}$ monocristallin pour différentes amplitudes de déformation en fonction de la déformation plastique cumulée [44].

[Cyclic hardening curves of $\mathrm{Cu}$ single crystals at different strain amplitudes as a function of the cumulative plastic strain [44].]

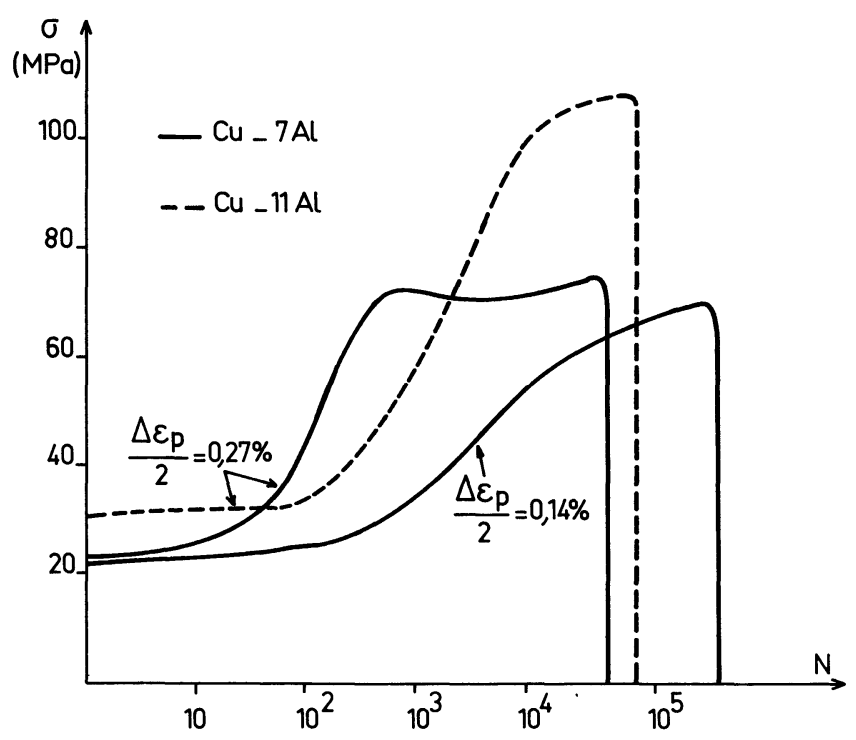

Fig. 7. - Courbes de consolidation cyclique d'alliages $\mathrm{Cu}-\mathrm{Al}$ monocristallins d'après [46].

[Cyclic hardening curves of single crystal $\mathrm{Cu}-\mathrm{Al}$ alloys, from [46].]

dans le cas de certains alliages de la prédéformation $[44,48-50]$. La relation $\Delta \sigma_{\mathrm{s}}\left(\Delta \varepsilon_{\mathrm{p}}\right)$ est souvent représentée par une loi en puissance :

$$
\Delta \sigma_{\mathrm{s}} / 2=K\left(\Delta \varepsilon_{\mathrm{p}} / 2\right)^{n},
$$

loi qui n'est valable en général que pour des matériaux polycristallins sollicités à $\Delta \varepsilon_{\mathrm{p}}$ élevé. En effet pour des monocristaux de métaux purs la contrainte de saturation présente la particularité d'être constante dans une large gamme de déformation plastique (gamme $\mathbf{B}$, cf. Fig. 8). Pour plusieurs matériaux les courbes de

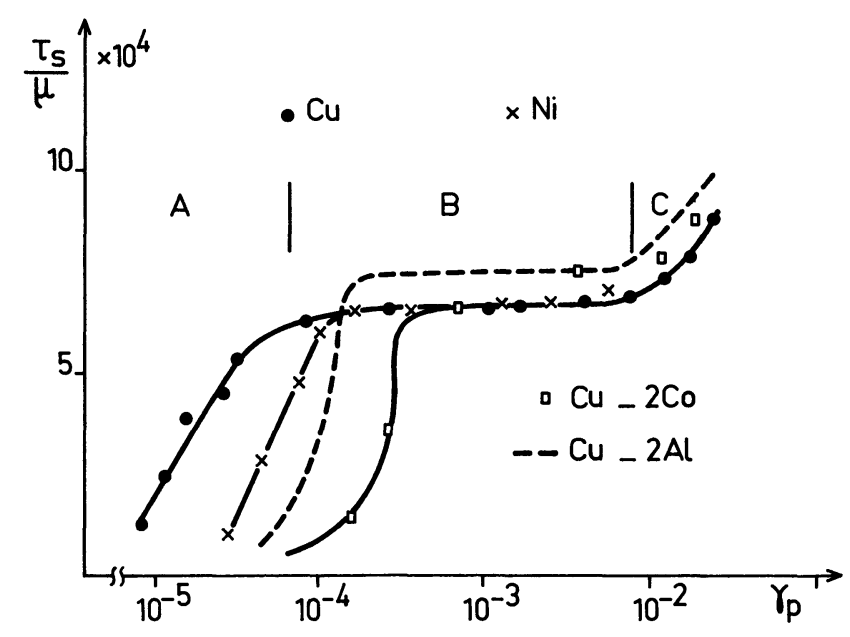

Fig. 8. - Courbes d'écrouissage cyclique de monocristaux de métaux purs et alliés $(\mathrm{Cu}, \mathrm{Ni}, \mathrm{Cu}-2 \% \mathrm{Al}, \mathrm{Cu}-2 \% \mathrm{Co})$, d'après [44 et 68].

[Saturation stress-strain curves of single crystal metals and alloys $(\mathrm{Cu}, \mathrm{Ni}, \mathrm{Cu}-2 \% \mathrm{Al}, \mathrm{Cu}-2 \% \mathrm{Co})$, from [44 and 68].]

contraintes normalisées $\left(\tau_{\mathrm{s}} / \mu\right)$ en fonction de l'amplitude de cisaillement $\gamma_{\mathrm{p}}$ (Fig. 8) présentent un plateau, à $\tau_{\mathrm{s}} / \mu$ constant, qui s'étend de $10^{-4}<\gamma_{\mathrm{p}}<10^{-2}$. Par contre à faible amplitude (gamme $\mathrm{A}$ ) ou à forte amplitude (gamme C) la contrainte de saturation croît avec l'amplitude de déformation. La présence de ce plateau est due à la localisation de la déformation plastique dans des bandes de glissement persistantes (BGP), phénomène particulier à la déformation cyclique et décrit en détail dans la partie (II). Les influences respectives de la température $[28,51]$ et de la vitesse de déformation y sont également décrites.

La boucle d'hystérésis. Les courbes précédentes sont obtenues à partir des valeurs extrêmes $\left(\sigma_{\max }\right.$, $\left.\varepsilon_{\mathrm{pmax}}\right)$ des boucles d'hystérésis lors d'une sollicitation cyclique. Or, souvent, la forme de la boucle (i.e. son caractère pointu ou rectangulaire) qui dépend de $\Delta \varepsilon_{\mathrm{p}}$ évolue également en cours de cyclage. Cette évolution peut être corrélée avec l'évolution mécanique et microstructurale due à la déformation cyclique. Par exemple, pour des monocristaux de $\mathrm{Cu}$ sollicités à différentes amplitudes de déformation, la figure 9 (d'après Mughrabi [44]) présente la variation du paramètre $V_{\mathrm{H}}$ (le rapport de l'aire de la boucle à celle du rectangle circonscrit) en fonction de $\gamma_{\text {p cum }}$. Dans la gamme A, $V_{\mathrm{H}}$ décroît au cours du premier stade et se stabilise à saturation. Dans la gamme $\mathrm{B}, V_{\mathrm{H}}$ décroît jusqu'à un minimum, puis croît jusqu'à sa valeur à saturation, elle-même croissante avec la déformation. Ce minimum de $V_{\mathrm{H}}$ apparaît à une déformation cumulée $(\$ 10)$ et une contrainte pour laquelle les premières BGP apparaissent en début de saturation [44, 52]. Dans la gamme C, les BGP apparaissent dès les premiers cycles; il n'y a plus de minimum. D'après Mecke et al. [53] le minimum de $V_{\mathrm{H}}$ est en fait constitué d'une série d'oscillations. Dans $\mathrm{Cu}-\mathrm{Al}$, où on retrouve ce 


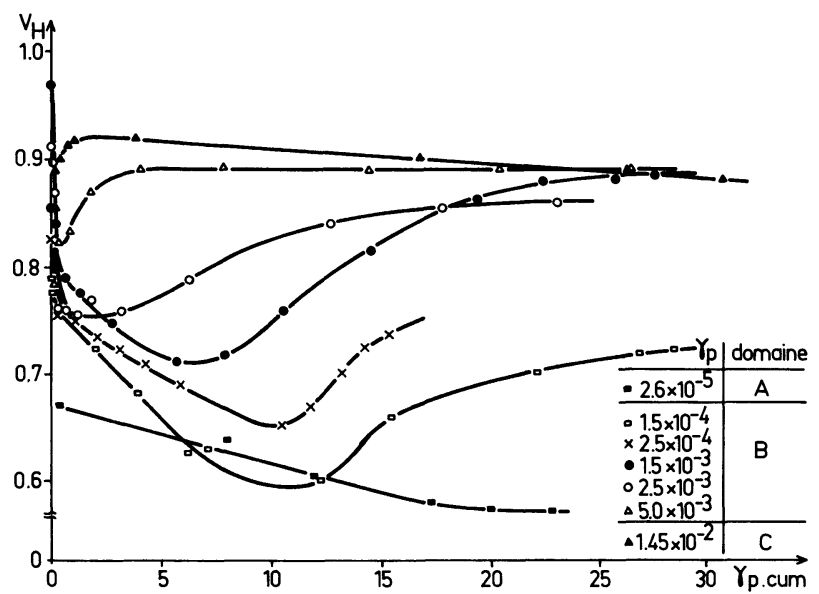

Fig. 9. - Variation dans le cuivre monocristallin du paramètre $V_{\mathrm{H}}$ décrivant la forme des boucles d'hystérésis en fonction de $\gamma_{\mathrm{p} \text { cum }}$, pour différentes valeurs de $\gamma_{\mathrm{p}}$ (les domaines $\mathrm{A}, \mathrm{B}, \mathrm{C}$ correspondent à la définition de la figure 8) [44].

[The variation of the hysteresis loop shape parameter $V_{H}$ with $\gamma_{\mathrm{pcum}}$ for different values of $\gamma_{\mathrm{p}}$ in single crystal $\mathrm{Cu}$ (the regions $\mathrm{A}, \mathrm{B}$ and $\mathrm{C}$ correspond to the definitions of figure 8 ) [44].]

phénomène [46], chaque oscillation correspond à l'émergence d'une nouvelle bande de glissement.

\subsubsection{Evolution de la microstructure de dislocations. -} Dans ce paragraphe, nous analysons l'évolution microstructurale jusqu'à l'établissement de la saturation. Ceci nous amènera à définir brièvement cet état de saturation à partir du modèle simple de Winter.

Observations en surface. Lorsque ces observations sont menées avec une résolution suffisante, elles permettent d'étudier la localisation du glissement et ses variations en fonction de $\gamma_{p}, \gamma_{p, c u m}$ et de la nature du matériau. Très schématiquement, on observe deux types de comportements lorsque l'EDE varie : à faible EDE, par exemple pour des monocristaux de $\mathrm{Cu}-\mathrm{Al}$ orientés en glissement simple et comportant de 7 à $16 \%$ atomiques d'aluminium, les traces de glissement primaires, très hétérogènes, apparaissent dès les premiers cycles, puis se densifient progressivement jusqu'à la rupture [46]. Par contraste, dans les C.F.C. purs ou faiblement alliés, la déformation tend à se localiser sous forme de bandes de glissement persistantes dont la géométrie peut varier suivant le matériau. Ainsi, leur épaisseur est de $1 \mu \mathrm{m}$ dans le cuivre, mais de seulement $0,1 \mu \mathrm{m}$ dans les alliages $\mathrm{Al}-\mathrm{Cu}$ à précipités. Dans le cas du cuivre monocristallin, qui a été le plus étudié, les observations optiques mettent en évidence les propriétés suivantes, également valables pour le nickel [54] : les BGP s'associent en « macrobandes » de glissement primaire, de quelques dizaines à quelques centaines de $\mu \mathrm{m}$ de large, et traversant tout le cristal [55]. Elles conduisent globalement à un gonflement local de l'échantillon. Comme le montre la figure 10 , le comportement de ces macrobandes est

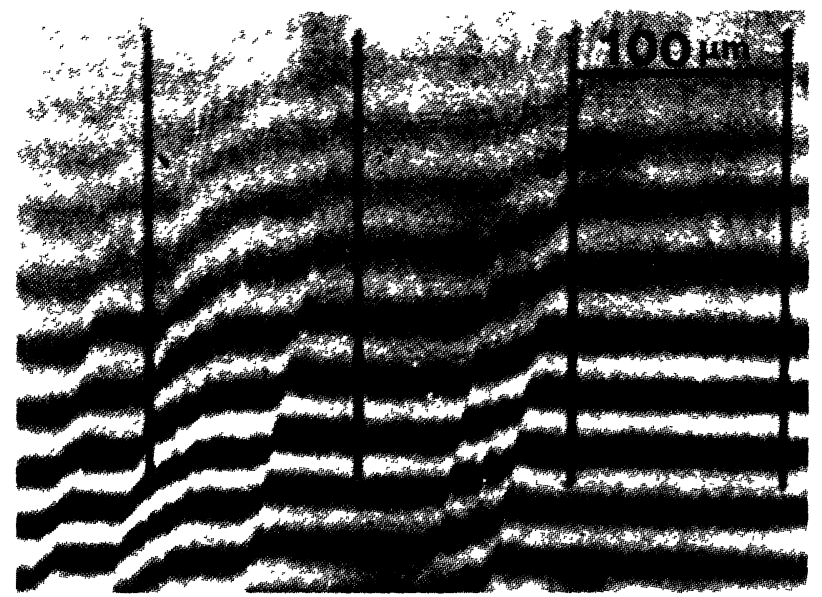

a)

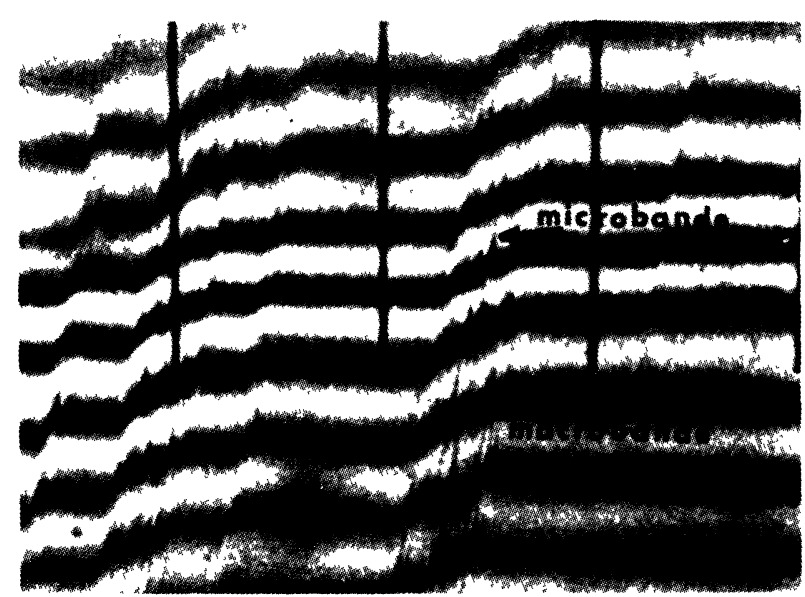

b)

Fig. 10. - Observations optiques montrant, au cours d'un petit nombre de cycles effectués dans le domaine $\mathrm{B}$, la réversibilité parfaite des macrobandes (mesurable par le décalage des franges de part et d'autre) et l'irréversibilité des microbandes situées à l'intérieur (BGP) [55]. a) $\frac{1}{4}$ cycle ; b) $10 \frac{1}{4}$ cycles.

[Interference contrast micrographs showing during a few cycles in region $B$, the perfect reversibility of the macrobands (as measured by the fringe displacements) and the irreversibility of the microbands (PSB) within the larger bands [55]. a) $\frac{1}{4}$ cycle ; b) $10 \frac{1}{4}$ cycles.]

parfaitement réversible au cours d'un petit nombre de cycles. Il n'en va pas de même pour les microbandes qui se trouvent à l'intérieur, comme le montre également la figure 10. Ces mesures mettent donc en évidence une irréversibilité locale de la déformation cyclique.

Le modèle de Winter. Les observations de surface montrent qu'à saturation, la fraction de la surface totale couverte par les BGP est proportionnelle à l'amplitude $\gamma_{\mathbf{p}}$. Puisque les BGP traversent tout le cristal, l'examen de la surface donne une bonne approximation de la fraction volumique, $f$, occupée par les BGP à saturation, la fraction non déformée 
de la matrice, $1-f$, constituant la matrice [47]. Cette mesure est d'autant plus simple et justifiée que $f$ est faible; en effet, à forte amplitude les BGP ne sont plus isolées mais groupées en macrobandes (cf. Fig. 10) et il devient difficile de juger de leur activité. Le matériau formé de la matrice (M) et des BGP se comporte donc comme un matériau composite. Une première approche élaborée par Winter [48], puis Finney et Laird [55], établit une analogie avec le comportement d'un mélange de deux phases. On peut ainsi écrire une loi de mélange [48] :

$$
\gamma_{\mathrm{p}}=f \gamma_{\mathrm{p}, \mathrm{BGP}}+(1-f) \gamma_{\mathrm{P}, \mathrm{M}}
$$

où $\gamma_{\mathrm{p}, \mathrm{i}}$ est la déformation plastique de la phase i. Cette relation étant vérifiée expérimentalement, il en résulte que $\gamma_{\mathrm{p}, \mathrm{M}}$ et $\gamma_{\mathrm{p}, \mathrm{BGP}}$ sont des constantes. Le matériau s'adapte donc à l'amplitude de déformation imposée $\gamma_{\mathrm{p}}$ en ajustant sa fraction volumique de BGP. Toutefois, lorsqu'on réduit l'amplitude après saturation, les BGP formées ne disparaissent pas mais demeurent actives.

En extrapolant aux valeurs limites, on obtient $\gamma_{\mathrm{p}}=\gamma_{\mathrm{p}, \mathrm{M}} \sim 10^{-4}$ pour $f=0$, et pour $f=1, \gamma_{\mathrm{p}}=$ $\gamma_{\mathrm{p}, \mathrm{BGP}} \sim 10^{-2}$. Ces valeurs correspondent assez bien aux limites du plateau de saturation pour le cuivre et le nickel $[44,48,55]$. Bien que la durée d'activité des BGP ne soit pas illimitée ce type d'approche reste d'une grande utilité car il établit un premier lien entre les conditions de sollicitation et les caractéristiques microstructurales.

Configuration des dislocations jusqu'à saturation. Nous allons maintenant examiner plus en détail la formation de la phase matrice. L'essentiel de ces résultats a été obtenu sur le cuivre, par microscopie électronique, ou études de surfaces par figures d'attaque. En règle générale il se forme une structure hétérogène dont l'échelle décroît au cours du cyclage, et dont l'allure dépend fortement de $\gamma_{\mathrm{p}}$. Basinski et al. [34] distinguent trois stades qui se succèdent sans transition brutale pour des amplitudes de déformation cumulée croissantes. Pendant les premiers cycles, les arrangements de dislocations, parallèles au plan de glissement et formant des enchevêtrements au milieu d'une structure peu dense, sont assez semblables à ceux obtenus dans le stade I de la déformation monotone. Le stade II est caractérisé par la formation de murs de dislocations dans le plan de glissement dévié, tandis que les traces de glissement deviennent fines et quasi-réversibles. Ceci indique que la microstructure commence à s'ajuster à la sollicitation imposée. Il suffit en effet d'une augmentation de $\gamma_{p}$ de $5 \%$ en cours d'essai pour obtenir des traces de glissement larges et irréversibles. Au cours du stade III, les murs se fragmentent en veines, conduisant à la structure matrice, typique du stade de saturation (cf. II, § 1.2). Contrairement au stade I, les structures des stades II et III sont typiques de la déformation cyclique.

Ces stades ne sont pas discernables sur les courbes de déformation cyclique en raison de leur caractère progressif ; les études menées sur le nickel [53] montrent de plus une forte influence de $\gamma_{p}$ : pour les valeurs correspondant au début du plateau de saturation cyclique, il se forme assez rapidement une structure de veines semblable à la structure matrice caractéristique de la saturation. Lorsque $\gamma_{p}$ augmente, cette structure tend à occuper une plus faible fraction volumique et à devenir moins dense. Lorsque les premières BGP apparaissent, et jusqu'au début du palier de saturation elle continue à évoluer. En vue d'une modélisation de ce comportement, on n'en retient que les éléments essentiels : la microstructure est essentiellement hétérogène; elle est constituée d'enchevêtrements, de veines (cf. Fig. II.1) formées de dipôles à caractère coin et qui se densifient au cours du cyclage. Ce sont donc là des structures peu déformables et qui évoluent de manière à toujours se trouver au voisinage de l'instabilité à la contrainte maximale de chaque cycle (cf. ci-dessous.). Dans les interstices ou canaux entre les veines, par contre, les dislocations vis peuvent évoluer librement.

Instabilités $d u$ durcissement cyclique : "strain bursts ». Les expériences de Neumann [56] mettent en évidence le fait que les sous-structures créées au cours de la consolidation, et qui à saturation vont donner naissance à la matrice et aux BGP, peuvent subir des transformations brutales en cours de cyclage.

Des monocristaux de cuivre sont déformés en fatigue avec une contrainte maximale par cycle qui croît linéairement avec le temps, jusqu'à une valeur de saturation de $32 \mathrm{MPa}$, atteinte au bout d'un nombre variable de cycles $N\left(250<N<10^{6}\right)$. Si $N$ est faible $\left(<2 \times 10^{3}\right)$, les faciès de surface sont ceux caractéristiques des fortes amplitudes de déformation par cycle; BGP, intrusions et extrusions sont présentes dès la saturation. A forte valeur de $N\left(>5 \times 10^{3}\right)$, en revanche, la déformation est extrêmement irrégulière comme le montre la figure 11. Bien que localisées dans le temps (et sur une durée de $\sim 50$ cycles) ces instabilités correspondent à une déformation répartie sur tout l'échantillon avec une bonne réversibilité (pas de reliefs de surface). Ce comportement est également observé sur des monocristaux d'aluminium et de magnésium, mais disparaît sur polycristaux [56].

L'interprétation microstructurale proposée par Neumann explique ces résultats par l'instabilité des configurations de dipôles présentes dans la matrice : l'élévation de contrainte dissocie ces dipôles et les dislocations ainsi libérées propagent l'instabilité à travers la microstructure par un processus d'avalanche. Si $N$ est faible, l'émergence en surface de ces dislocations rend le phénomène irréversible. $\mathrm{Si} N$ est fort, les dipôles peuvent se reformer avant d'émerger en surface, ce qui conduit à une bonne réversibilité de la déformation car il n'y a pas perte de dislocations d'un signe donné. Ce « repiégeage " des dislocations correspond alors au durcissement observé entre deux avalanches (cf. Fig. 11). L'idée d'une instabilité des 


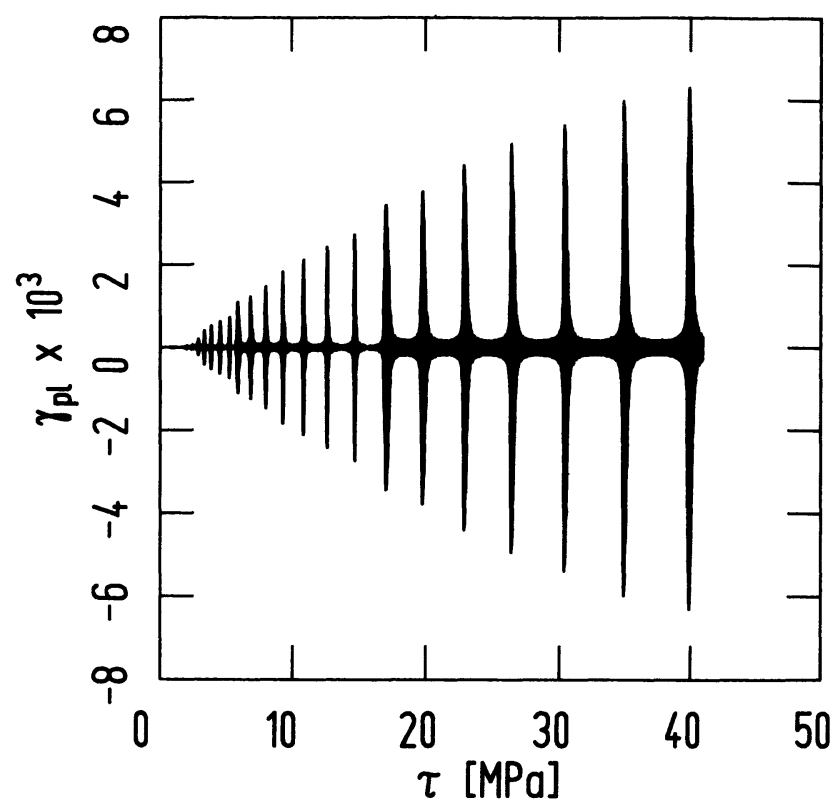

Fig. 11. - Instabilités périodiques de déformation d'un monocristal de cuivre cyclé à contrainte maximale croissante [56b].

[Periodic deformation instabilities in a copper single crystal cycled with an increasing maximum stress [56b]]

structures de dipôles a été reprise par divers auteurs en vue d'expliquer la formation des BGP, bien que ce ne soit pas là le but initial du modèle de Neumann [57].

La formation des BGP. La question se pose de savoir par quel processus la structure irrégulière de la matrice peut donner naissance aux configurations hautement organisées des BGP (cf. II, § 1.2). A ce jour, les réponses restent incomplètes. Les résultats de Neumann montrent bien que, lors de la consolidation, la matrice évolue tout en restant à la limite de la stabilité. Au-delà d'une valeur critique de la contrainte (à $\gamma_{\mathrm{p}}$ imposé) la structure de la matrice ne peut plus accommoder la déformation et se déstabilise. Kuhlmann-Wilsdorf et Laird [58] ainsi que Winter [59], ont proposé par analogie avec les instabilités de Neumann un mécanisme qui semble généralement accepté [60] : la partie externe des veines de la matrice est " durcie " par ses interactions avec les dislocations se déplaçant dans les canaux. La partie interne se trouve alors dans l'impossibilité d'évoluer car elle n'échange plus de dislocations avec l'extérieur. C'est donc l'effondrement interne des veines de la matrice qui conduirait à la formation des BGP dont les murs devraient correspondre à la partie externe des veines. Il existe d'autres modèles analogues [61], mais la réalité est sans doute plus complexe comme l'a montré Mughrabi (résultats non publiés). Sur le plan quantitatif, on dispose de mesures de la contrainte d'apparition des premières BGP en surface dans le cuivre [44], $\tau_{\mathrm{n}}$, qui décroît linéairement avec $\gamma_{\mathrm{p}}$. Si $\gamma_{\mathrm{p}}$ est faible $\left(<10^{-3}\right)$, les BGP, en faible fraction volumique, apparaissent au début de la saturation et $\tau_{\mathrm{n}}=\tau_{\mathrm{s}} \sim$ $28 \mathrm{MPa}$. A fort $\gamma_{\mathrm{p}}$, la matrice est moins bien formée et la propagation des BGP en est facilitée. Ceci explique que pour $\gamma_{\mathrm{p}} \simeq 10^{-2}, \tau_{\mathrm{n}}$ décroît jusqu'à $10 \mathrm{MPa}$. On peut alors considérer que la structure de la matrice tend à se rapprocher de celle des BGP, les deux structures se confondant en fin de palier de saturation lorsque $f=1$.

Modèles de consolidation cyclique. Au cours de la consolidation, les évolutions de la microstructure et de la boucle d'hystérésis sont relativement complexes. Divers modèles tentent de retrouver la forme de ces boucles d'hystérésis à partir des observations microstructurales [62]. Certains auteurs [63-64] adoptent une autre démarche : la contrainte maximale et la contrainte d'écoulement sont décomposées en une contrainte de friction et une contrainte interne, qui sont analysées en fonction de la déformation cumulée. Il semble toutefois difficile de donner un sens physique à ces résultats en termes de microstructures. L'approche inverse qui consiste à partir d'une vision simplifiée du comportement microscopique, mais en tenant compte en particulier de l'hétérogénéité des microstructures, semble plus prometteuse au vu des résultats obtenus.

Compte-tenu des observations rapportées ci-dessus, il semble qu'il y ait accord sur plusieurs points [60]. A l'intérieur des canaux les dislocations vis peuvent s'annihiler par glissement dévié. Ceci constitue une cause d'irréversibilité puisqu'une dislocation vis donnée a une probabilité non négligeable de ne jamais retourner à son lieu de création. Lors de leur mouvement les dislocations vis déposent des segments coins dans les amas denses, contribuant ainsi à leur densification (cf. Fig. 12); la longueur de ces segments coins est directement liée à l'irréversibilité. Comme on le verra au $\S$ II. 1, des considérations semblables ont été développées pour l'étude de la saturation.

On définit alors deux contraintes critiques :

$$
\tau_{\mathrm{v}}=\mu b / 4 \pi s, \text { et } \tau_{\mathrm{c}}=\mu b / 8 \pi(1-v) s,
$$

où $s$ est l'espacement entre plans de glissement. $\tau_{v}$ est la contrainte critique de croisement pour deux vis et $\tau_{\mathrm{c}}$ son analogue pour les coins; $\tau_{\mathrm{c}}$ peut également être considérée comme la contrainte d'instabilité d'un dipôle. Comme la structure de dipôles se trouve toujours au voisinage de l'instabilité, leur largeur moyenne va décroître au cours de la consolidation, par déstabilisation des plus larges d'entre eux. Une autre contrainte critique est la contrainte d'Orowan, pour la courbure d'une boucle entre deux " paquets " de dipôles, comme illustré par la figure 12 :

$$
\tau_{0}=\alpha \mu b / l
$$

où $l$ est la largeur des canaux, qui diminue avec la déformation cumulée.

A ce jour, le modèle de consolidation le plus élaboré 


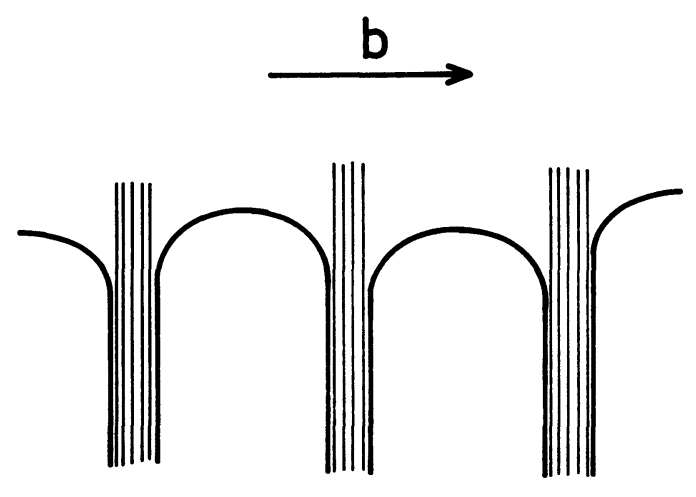

(a)

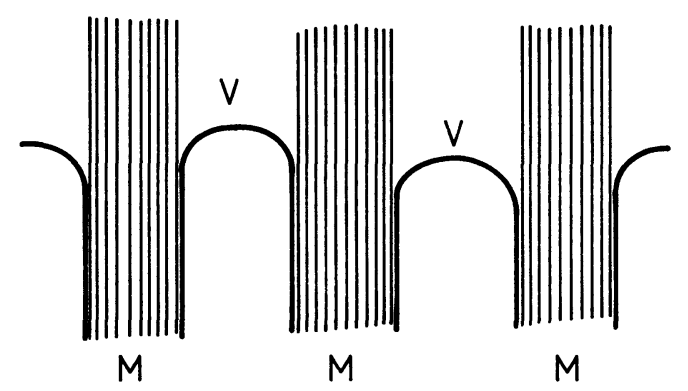

$\mathrm{V}=\mathrm{Vis}$ $M=$ Mur

(b)

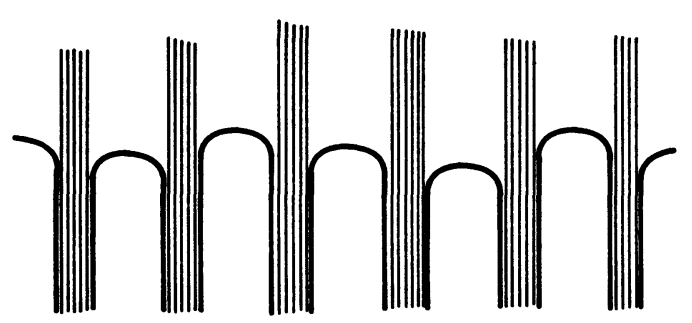

(c)

Fig. 12. - Schéma de l'évolution microstructurale au cours de l'écrouissage cyclique. Les dislocations vis sont courbées entre des amas de dipôles coins dont l'espacement décroît lorsque la contrainte croît (le plan de glissement est parallèle au plan du schéma) [60].

[Schematic of the microstructural change during cyclic hardening. The screw dislocations are curved against the edge dipole clusters whose spacing decreases as the stress increases (the slip plane is parallel to the plane of the diagram) [60].]

est celui récemment proposé par Pedersen et Winter [62], dans lequel le cristal est considéré comme un composite formé d'une phase "dure " (les paquets de dipôles) et d'une phase déformable, les canaux. Ceci apparaît clairement du fait que les contraintes mesurées en cours de déformation cyclique sont supérieures (d'un facteur 3 ou 4) à la contrainte d'écoulement $\tau_{0}$ de la phase déformable. En ce qui concerne les contraintes internes, que l'on peut mettre en évidence par l'analyse des boucles d'hystérésis, elles résultent alors des conditions de compatibilité entre ces deux phases.

Le calcul de la consolidation est alors effectué en considérant les paquets de dipôles comme une inclu- sion indéformable (ce qui n'est pas tout à fait le cas puisque cette structure évolue) à l'intérieur d'une phase déformable. Les résultats obtenus sont en bon accord avec les mesures de Basinski et al. [34] jusqu'aux valeurs de la déformation cumulée correspondant à la formation des BGP.

Ce type d'approche permettra peut-être d'aboutir dans l'avenir à une prédiction de la forme des boucles d'hystérésis. A l'heure actuelle, cependant, la fraction volumique des « inclusions » et son évolution en fonction de $\gamma_{p}$ et de $\gamma_{p \text {,cum }}$ ne peuvent être calculées et sont obtenues à partir des observations en microscopie électronique.

Des modèles semblables, quoique plus élémentaires, ont été utilisés pour décrire la forme des boucles d'hystérésis au cours de la saturation $[57,65]$.

3.2 Cas des métaux et alliages C.C. - Nous décrivons d'abord le comportement mécanique des métaux et alliages C.C., puis nous montrons comment il peut être interprété à partir des propriétés des dislocations dans ces structures.

3.2.1 Comportement mécanique. - Durcissement cyclique. Courbes d'écrouissage cyclique. - Les résultats obtenus sur des monocristaux de fer $\alpha$ sollicités à température ambiante et à une vitesse $\dot{\varepsilon} \simeq 5 \times$ $10^{-4} / \mathrm{s}$ [68], illustrent le comportement général des monocristaux C.C. dans le domaine "basse température " $\left.\left(T<T_{\mathrm{a}}\right): 1\right)$ Pour des amplitudes de déformation plastique élevées $\left(\geqslant 3 \times 10^{-3}\right)$, le durcissement cyclique est important et rapide; il est suivi par un état de saturation bien établi. 2) Pour des faibles amplitudes $\left(\Delta \varepsilon_{\mathrm{p}} / 2<10^{-3}\right)$ le durcissement est très réduit, contrairement au caś du cuivre ou du nickel. Ce faible durcissement produit des discontinuités dans les courbes d'écrouissage cyclique qui présentent alors trois stades. C'est ce que montre la figure 13 pour des monocristaux de Fe-6 Si [69], à $\dot{\varepsilon}=2 \times 10^{-3} / \mathrm{s}$. Une telle absence de durcissement à $\Delta \varepsilon_{\mathrm{p}} / 2<10^{-3}$ a été également constatée sur des monocristaux de niobium [70] et semble caractéristique des cristaux C.C. sollicités à $T<T_{\mathrm{a}}$. Nous avons déjà mentionné l'existence de lois d'écrouissage cyclique empiriques comme celle donnée par l'expression (2). Certains auteurs ont montré qu'une telle équation mécanique pouvait servir à la prédiction des durées de vie en fatigue [71]. Cependant, on constate ici qu'une seule équation mécanique ne peut en aucun cas décrire le comportement des C.C. sollicités à basse température. Ajoutons enfin qu'un tel phénomène est également observé sur polycristaux [72].

L'influence de la vitesse de déformation apparaît également sur la figure 13. Aux faibles vitesses $\left(\sim 10^{-5} / \mathrm{s}\right)$ les courbes d'écrouissage sont plus conventionnelles et ne présentent pas de discontinuités. Les contraintes sont plus faibles, mais le taux de consolidation est plus élevé, et ceci quelle que soit l'amplitude de la déformation plastique. Notons que certains alliages peuvent présenter un durcissement 


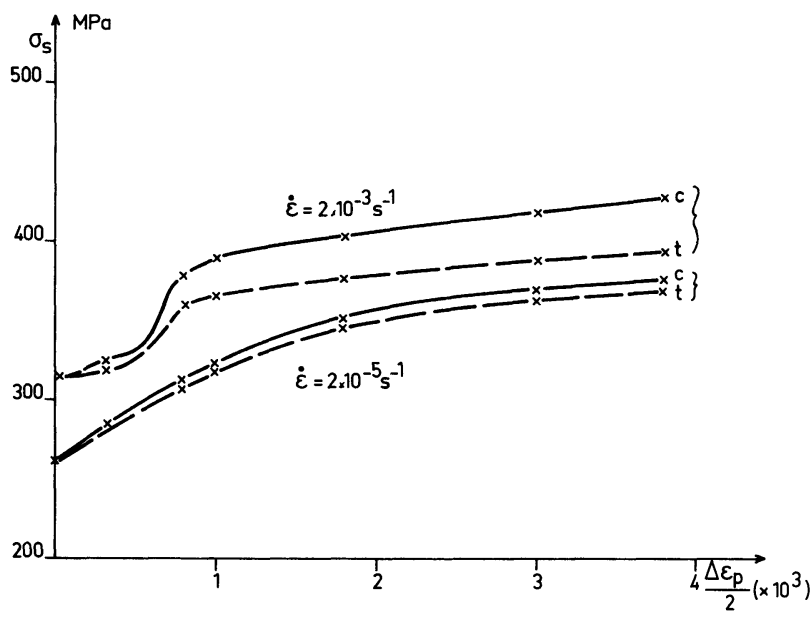

Fig. 13. - Influence de $\dot{\varepsilon}$ sur les courbes d'écrouissage cyclique de cristaux [001] $\mathrm{Fe}-6 \% \mathrm{Si}$ à température ambiante (c $=$ compression, $\mathrm{t}=$ traction) [69].

[The influence of $\dot{\varepsilon}$ on the saturation stress-strain curves of [001] $\mathrm{Fe}-6 \% \mathrm{Si}$ crystals at room temperature (c $=$ compression, $\mathrm{t}=$ tension) [69].]

cyclique, même à l'état recuit, pour de faibles amplitudes de déformation imposée $[69,73]$. Enfin, les essais de changement de vitesse de déformation (cf. $\S 2.2$ ) permettent de déterminer la contrainte effective, $\sigma^{*}$, et interne, $\sigma_{\mathrm{i}}$. Ainsi, pour des monocristaux de fer [74], de niobium [70], de Fe-6 Si et Fe-26 Cr-1 Mo [75] sollicités à température ambiante, on observe que : 1) l'augmentation de la contrainte d'écoulement pendant le durcissement cyclique est due à l'augmentation de $\sigma_{\mathrm{i}}$, comme en déformation monotone, 2) la contrainte effective $\sigma^{*}$ est la composante principale de la contrainte d'écoulement lorsque $\dot{\varepsilon}$ est élevé $\left(>10^{-3} / \mathrm{s}\right)$ alors que $\sigma_{\mathrm{i}}$ est nettement supérieur à $\sigma^{*}$ aux faibles valeurs de $\dot{\varepsilon}\left(<10^{-5} / \mathrm{s}\right)$.

Asymétrie de comportement entre traction et compression. - Les deux phénoménes décrits ci-dessous découlent directement des effets de structure de cour des dislocations sur la géométrie du glissement (cf. $\S 2.2 .1$ ).

a) Asymétrie des contraintes : lors d'essais à amplitude de déformation imposée (identique en traction et en compression) certaines orientations monocristallines manifestent une asymétrie de contrainte entre traction et compression, comme cela apparaît sur la figure 13. Cet effet peut avoir deux origines [75, 76] : 1) soit les systèmes de glissement diffèrent en traction et en compression, 2) soit le glissement se produit sur des systèmes $\{h k l\}\langle 111\rangle$, avec $\{h k l\} \neq$ $\{110\}$, pour lesquels les deux sens de sollicitation ne sont pas énergétiquement équivalents. Ce dernier cas correspond à celui des cristaux $\langle 001\rangle$ de $\mathrm{Fe}-6 \mathrm{Si}$ (cf. Fig. 13). Le glissement, qui a lieu sur des systèmes $\{112\}\langle 111\rangle$ est énergétiquement plus facile en traction (sens " maclage ") qu'en compression (sens "antimaclage »). Le signe de l'asymétrie de con- trainte $\Delta\left(\Delta(\%)=100\left(\sigma_{\text {comp. }}-\sigma_{\text {trac. }}\right) / \sigma_{\text {moyen }}\right)$ dépend de l'orientation cristallographique ; il peut être directement déterminé à partir de l'identification des systèmes de glissement [75].

L'asymétrie du glissement, typique des matériaux C.C. à basse température et qui conduit à une asymétrie de contrainte à déformation imposée [77-79] provoque une asymétrie de déformation (allongement ou rétrécissement des éprouvettes) à contrainte imposée $[69,76]$. Enfin, on note que l'asymétrie de contrainte est réduite aux faibles amplitudes de déformation (Fig. 13) et aux faibles vitesses de déformation (Fig. 14), et qu'elle est inexistante pour des cristaux se déformant exclusivement par glissement sur des plans $\{110\}$.

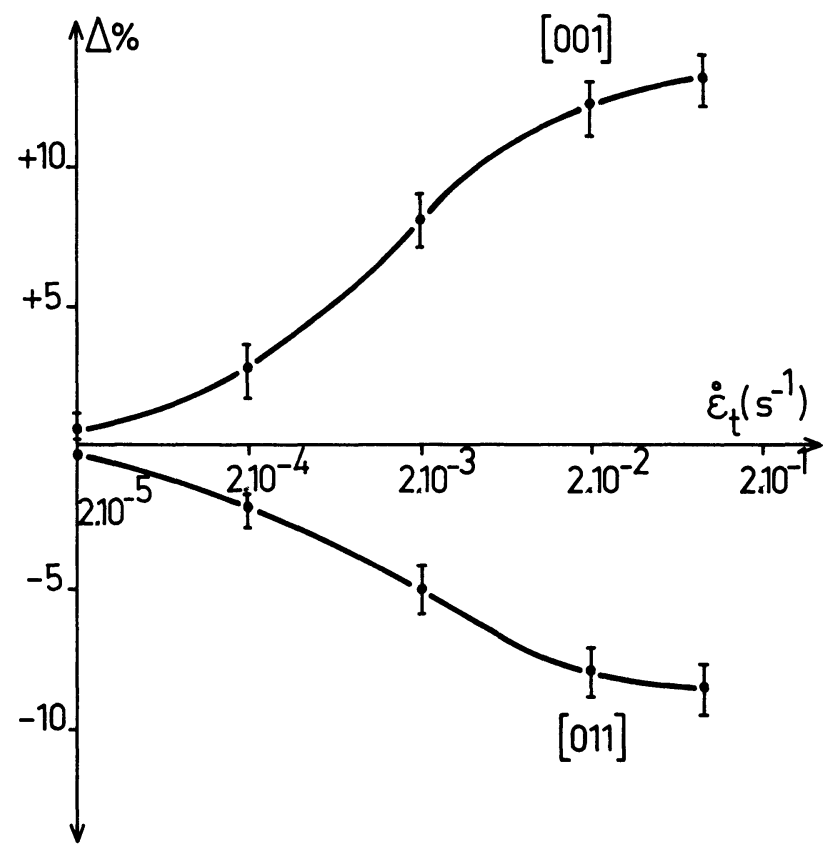

Fig. 14. - Influence de la vitesse de déformation sur l'asymétrie de contrainte $\Delta$ de cristaux [001] et [011] Fe-26 Cr-Mo déformés à $\Delta \varepsilon_{\mathrm{t}} / 2=6 \times 10^{-3}$ [75].

[The influence of the strain rate on the stress asymmetry $\Delta$ of [001] and [011] Fe-26 Cr-Mo crystals deformed at $\Delta \varepsilon_{t} / 2=$ $\left.6 \times 10^{-3}[75].\right]$

b) Changement de forme : lorsque les cristaux sont sollicités symétriquement en traction et en compression à déformation imposée, l'activation de systèmes différents suivant le sens de la sollicitation conduit à un déplacement irréversible de matière. Le changement de forme des éprouvettes qui en résulte est, certes, faible sur un cycle (typiquement $10^{-4}-10^{-3}$ ), mais devient important sur un grand nombre de cycles [69, 70, 80-82]. Ainsi, la section des éprouvettes, initialement circulaire et de diamètre $d_{0}$ devient elliptique (grand axe $: d_{\max }$, petit axe $: d_{\min }$ ). Le changement de forme mesuré par le rapport $d_{\max } / d_{0}$ est fonction de l'amplitude de la déformation (Fig. 15), de la vitesse de déformation et de la température (Fig. 16). De plus 


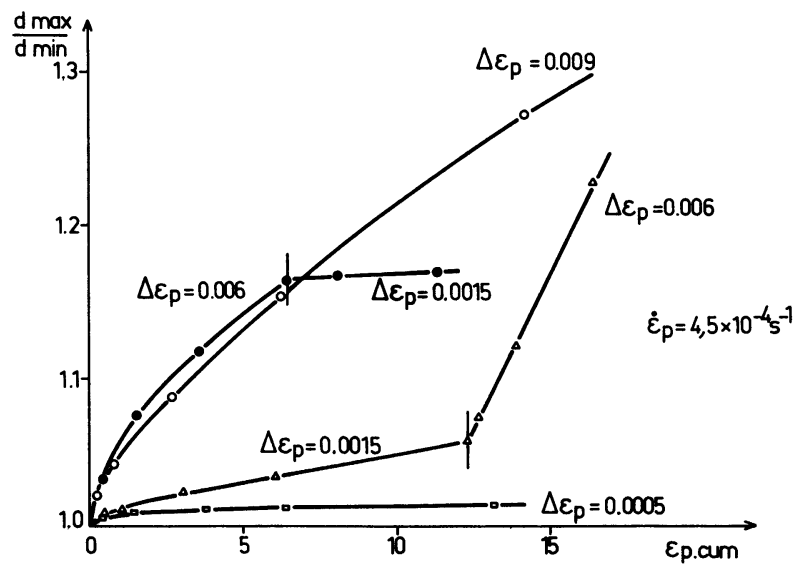

Fig. 15. - Influence de $\varepsilon_{\mathrm{pcum}}$ et de $\Delta \varepsilon_{\mathrm{p}}$ sur le changement de forme des monocristaux de $\mathrm{Fe} \alpha$ orientés pour glissement simple [68].

[The influence of $\varepsilon_{\mathrm{pcum}}$ and $\Delta \varepsilon_{\mathrm{p}}$ on the shape change of single slip oriented $\alpha \mathrm{Fe}$ crystals [68].]

pour Fe et Mo à la température ambiante, il est très faible aux petites amplitudes de déformation $\left(\Delta \varepsilon_{\mathrm{p}} \simeq\right.$ $\left.10^{-4}\right)$ ainsi qu'aux basses vitesses $\left(\dot{\varepsilon} \simeq 10^{-5} / \mathrm{s}\right)$. Pour une amplitude de déformation donnée, il augmente lorsque la température décroît.

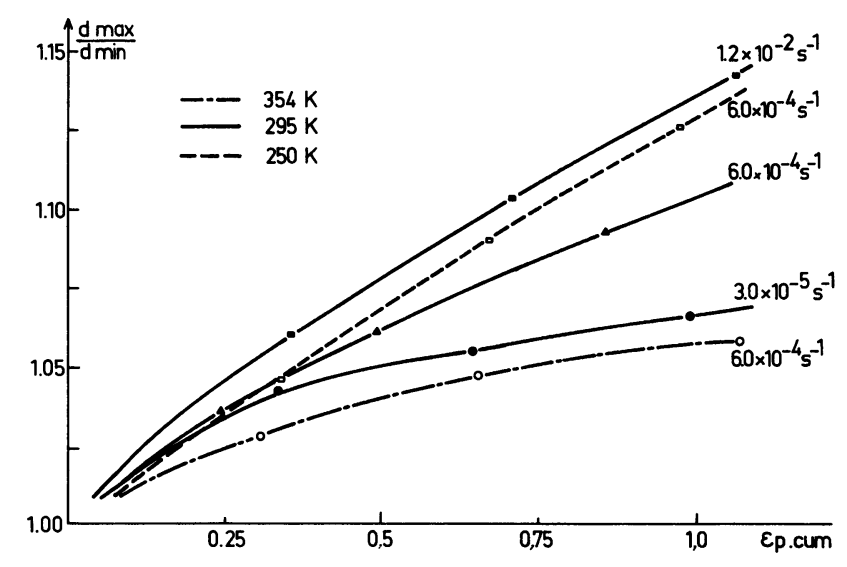

Fig. 16. - Influence de la température et de la vitesse de déformation sur l'évolution du changement de forme pour des cristaux de $\mathrm{Nb}$ orientés pour glissement simple [70].

[The influence of temperature and strain rate on the shape change of single slip oriented $\mathrm{Nb}$ crystals [70].]

Le changement de forme peut se produire sans qu'une asymétrie de contrainte soit observée lorsque de petites différences entre plans de glissement en compression et en traction sont constatées (par exemple autour de $\{110\})$, alors que l'asymétrie

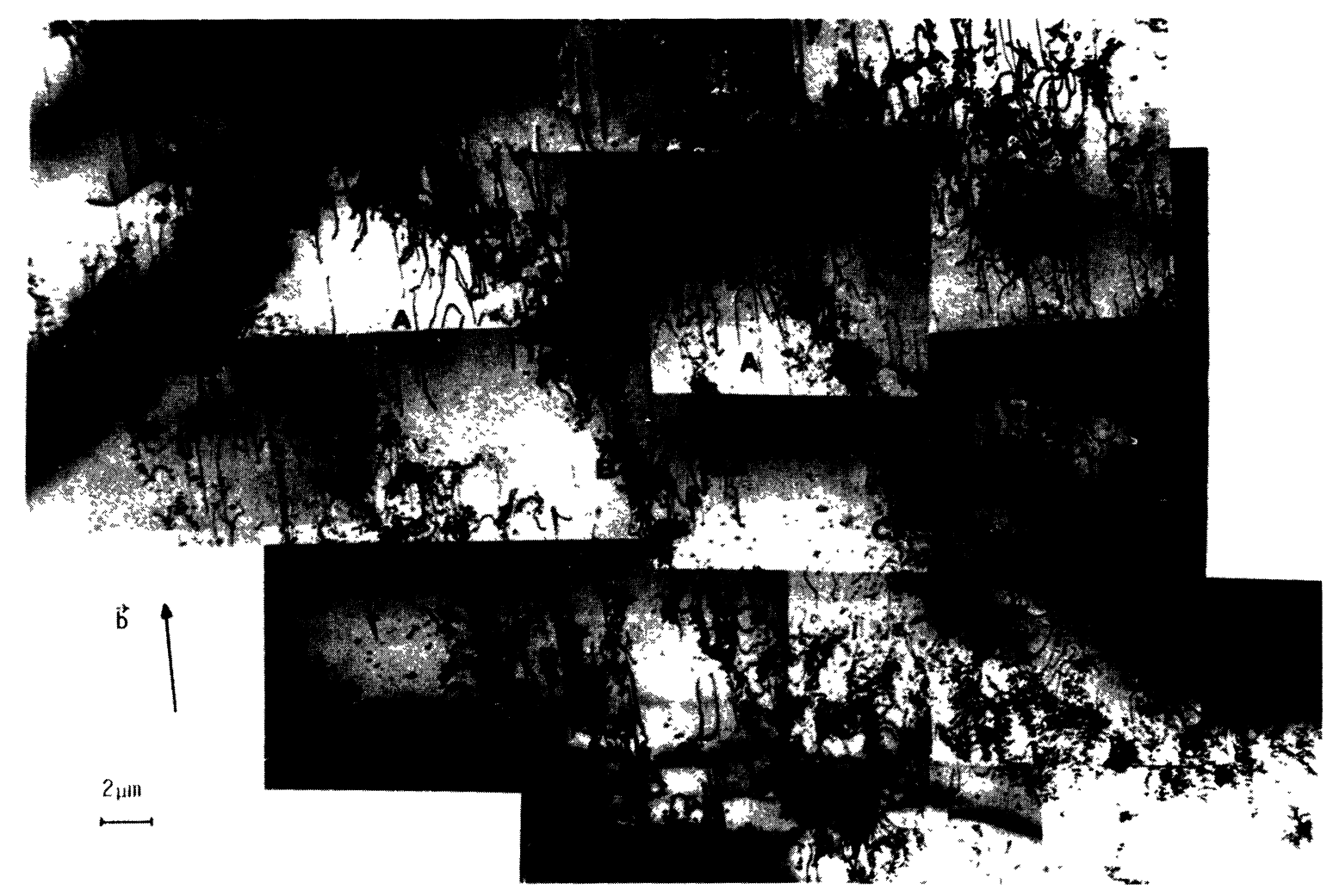

Fig. 17. - Arrangement des dislocations sur le plan (101) d'une éprouvette [1 149$] \mathrm{Fe}-26 \mathrm{Cr}$-Mo après 20 cycles à $\Delta \varepsilon_{\mathrm{t}} / 2=$ $6 \cdot 10^{-3}\left(\varepsilon_{\text {p cum }} \simeq 0,4\right)$ et $\dot{\varepsilon}_{\mathrm{t}}=2 \cdot 10^{-3} \mathrm{~s}^{-1}[88]$.

[Dislocation configurations on the (101) plane of a [1149] Fe-26 Cr-Mo crystal after 20 cycles at $\Delta \varepsilon_{\mathrm{t}} / 2=6 \times 10^{-3}\left(\varepsilon_{\mathrm{pcum}} \simeq\right.$ $0.4)$ and $\varepsilon_{\mathrm{t}}=2 \times 10^{-3} \mathrm{~s}^{-1}[88]$.] 
de contrainte a lieu sans changement de forme lorsqu'un seul plan de glissement, $\{112\}$ ou $\{h k l\} \neq$ $\{110\}$ est actif en traction et en compression [25]. Enfin, pour les monocristaux d'axes de haute symétrie comme $\langle 100\rangle$, qui peuvent présenter de fortes asymétries de contrainte, les changements de forme sont quasi-négligeables. L'influence de l'orientation cristallographique sur le taux de changement de forme est largement décrit par ailleurs [70].

3.2.2 Evolution de la microstructure. Interprétation du comportement mécanique. - Dans ce paragraphe nous décrivons les configurations de dislocations qui apparaissent au cours du durcissement cyclique avant que la déformation ne se localise (cette localisation sera étudiée en II) et nous interprétons les caractéristiques mécaniques précédemment exposées.

Influence de l'amplitude de la déformation plastique. L'évolution des sous-structures de dislocations formées en fatigue plastique aux très basses amplitudes $\left(10^{-3}\right)$, dans le régime « basses températures », n'a fait l'objet que de peu d'études; on ne peut guère citer que celle sur monocristaux de fer $\alpha$ sollicités à température ambiante et $\dot{\varepsilon}$ élevée [83, 84]. Même aux fortes valeurs de $\varepsilon_{\text {p,cum }}(\sim 100)$, l'augmentation de la densité de dislocations au cours du cyclage reste faible. De longs segments vis sont visibles en MET, ce qui confirme leur faible mobilité. La déformation cyclique est assurée par le mouvement quasi-réversible des dislocations coins. Les possibilités d'interaction et de multiplication des dislocations sont réduites, ce qui explique le taux de durcissement négligeable constaté précédemment dans ce cas.

Lorsque $\Delta \varepsilon_{\mathrm{p}}>10^{-3}$, l'arrangement des dislocations devient tout à fait différent. La figure 17 présente une vue générale de cet arrangement dans le plan de glissement d'un monocristal de $\mathrm{Fe}-26 \mathrm{Cr}$ orienté en glissement simple, et pour un état d'endommagement qui permet d'observer sur une même vue les différentes configurations de dislocations conduisant à la saturation. Au cours des premiers cycles, les dislocations de caractère essentiellement vis interagissent et leur densité augmente (présence de longs segments vis avec une forte densité de superdécrochements, cf. (A), Fig. 17). Grâce à l'annihilation par glissement dévié, des dipôles coins apparaissent et s'enchevêtrent pour former des multipôles (B). Par interaction, de petites boucles de dislocations appelées débris (C) se forment alors. Rapidement des amas de dislocations, formés essentiellement de multipôles et de débris se constituent (D). Entre les amas, des dislocations vis mobiles accommodent la déformation, leur densité diminuant progressivement. Pour franchir ces amas épars, les dislocations vis sont contraintes d'effectuer des doubles glissements déviés, provoquant la formation de plus en plus abondante de débris, ce qui conduit à une densification des amas. Ces nouveaux obstacles provoquent un accroissement de la contrainte interne, donc de la contrainte d'écoulement et conduisent à l'épaississement des amas et à leur transformation en parois puis en cellules. L'état de saturation est alors atteint. Il correspond à un durcissement presque négligeable, ce qui ne signifie nullement que la microstructure n'évolue plus. La saturation sera en fait donnée par un équilibre entre l'interaction dislocations-parois et la libération de dislocations à partir de ces parois. On trouvera par ailleurs d'autres études similaires, notamment sur monocristaux de $\mathrm{Fe} \alpha$ [83], de $\mathrm{Nb}$ [85] et de Fe-6 Si [86].

Influence de la vitesse de déformation et de la température. L'effet de la vitesse de déformation sur le type de dislocations mis en jeu au cours de la consolidation cyclique éclaire davantage, par rapport au cas des C.F.C., le cas des C.C. sollicités au voisinage de la température athermique $T_{\mathrm{a}}$. La figure 18 indique la nette différence entre les configurations obtenues à

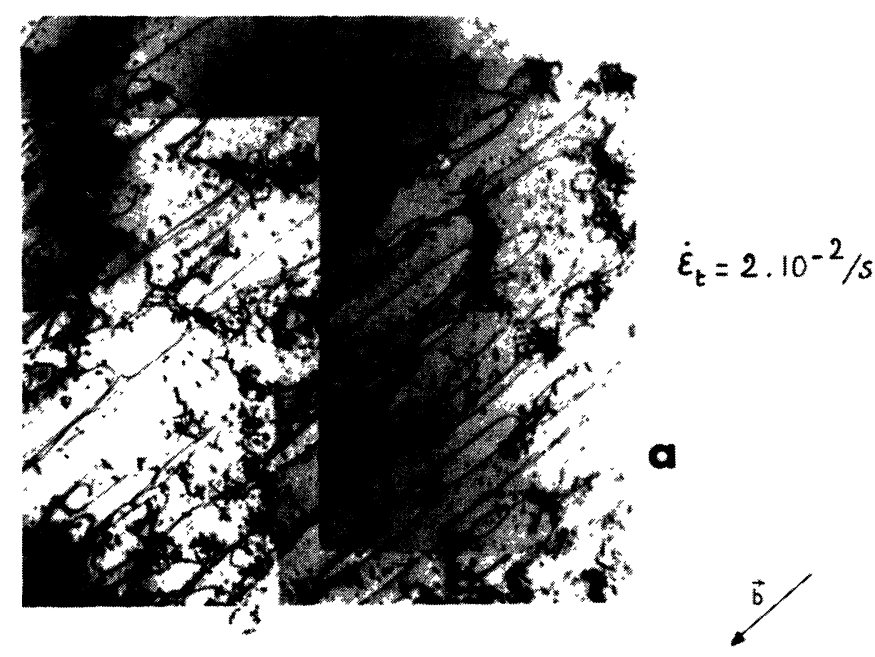

$\stackrel{1 . m}{\longrightarrow}$

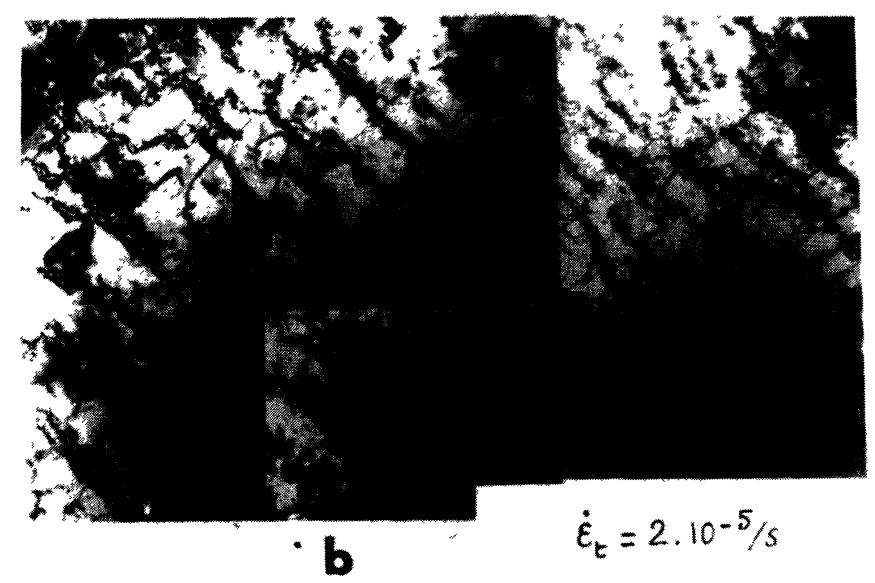

Fig. 18. - Influence de la vitesse de déformation sur l'arrangement des dislocations sur le plan $(\overline{2} 11)$ de cristaux [011] Fe-26 Cr-Mo déformés à $\Delta \varepsilon_{t} / 2=6 \cdot 10^{-3}$ pendant 10 cycles, (a) $\dot{\varepsilon}_{\mathrm{t}}=2 \times 10^{-2} \mathrm{~s}^{-1}$,(b) $\dot{\varepsilon}_{\mathrm{t}}=2 \times 10^{-5} \mathrm{~s}^{-1}$ [88].

[The influence of strain rate on the dislocation configurations on the ( $\overline{2} 11)$ plane of [011] Fe-26 Cr-Mo crystals deformed at $\Delta \varepsilon_{\mathrm{t}} / 2=6.10^{-3}$ for 10 cycles, (a) $\dot{\varepsilon}_{\mathrm{t}}=2 \times 10^{-2} \mathrm{~s}^{-1}$, (b) $\dot{\varepsilon}_{\mathrm{t}}=2 \times 10^{-5} \mathrm{~s}^{-1}[88]$.] 
forte et faible vitesse dans des monocristaux d'acier $\mathrm{Fe}-26 \mathrm{Cr}$ à température ambiante. A forte vitesse, on observe la présence de longues dislocations vis rigides et de quelques amas épars; le volume d'activation $V^{*}$ est de l'ordre de $20 b^{3}$, et $\sigma^{*}>\sigma_{\mathrm{i}}$. A faible vitesse la densité de dislocations est plus importante, et ces dislocations sont courbées et donc de caractère mixte $\left(V^{*} \sim 120 b^{3}\right.$ et $\left.\sigma_{\mathrm{i}}>\sigma^{*}\right)$. De plus, les amas sont déjà bien formés et alignés perpendiculairement au vecteur de Burgers. Le comportement «basse vitesse " est très proche de celui observé pour les C.F.C. Une telle influence de $\dot{\varepsilon}$ a été également constatée sur des monocristaux de fer $\alpha$ [87]. Enfin, l'influence de la température a pu être mise en évidence sur des monocristaux de Fe-6 $\mathrm{Si}$ [86] et de $\mathrm{Nb}$ [85] : comme dans tout processus activé, elle intervient en sens inverse de la vitesse de déformation.

Interprétation $d u$ comportement mécanique. Les résultats précédemment exposés permettent de définir deux domaines de comportement cyclique dans les métaux C.C. suivant les conditions de sollicitation.

a) Lorsque $T<T_{\mathrm{a}}$ (ce qui correspond à une basse température ou à une vitesse de déformation élevée, par exemple $\dot{\varepsilon}>10^{-3} / \mathrm{s}$ à $300 \mathrm{~K}$ pour le fer $\alpha$ ), la déformation est essentiellement contrôlée par des dislocations vis dont la forte rigidité est une conséquence de la forte friction de réseau. Ce régime de déformation est caractérisé par une forte asymétrie de comportement entre traction et compression due aux propriétés particulières du cœur des dislocations vis dans le réseau C.C. Ceci conduit aux effets d'asymétrie de contrainte et de changement de forme des cristaux. Enfin, les différents stades de la courbe d'écrouissage cyclique s'expliquent par une contribution croissante des dislocations vis à la déformation.

b) Lorsque $T>T_{\mathrm{a}}$ (température élevée, ou faible vitesse de déformation, par exemple $\dot{\varepsilon}<10^{-4}$ s pour le fer à $300 \mathrm{~K}$ ), sous l'effet de l'activation thermique les mobilités des dislocations vis et coins sont comparables. Les effets d'asymétrie sont alors négligeables et les densités de dislocations sont beaucoup plus élevées que précédemment. Le durcissement cyclique est prononcé même aux faibles valeurs de $\Delta \varepsilon_{\mathrm{p}}$, les trois stades observés à $T<T_{\mathrm{a}}$ sur les courbes d'écrouissage n'apparaissent plus. Le comportement se rapproche alors de celui des métaux C.F.C.

Notons pour terminer que, d'après ce qui précède, tout facteur qui intervient sur la différence de mobilités entre dislocations vis et coins influencera le régime de déformation, et donc le comportement mécanique des C.C. C'est notamment le cas des interstitiels comme le carbone dans le fer $\alpha$ [66], qui favorise ainsi un comportement du type haute température $\left(T>T_{\mathrm{a}}\right)$. Nous verrons dans la seconde partie (II) que la teneur en interstitiels influence également la localisation de la déformation plastique au cours du cyclage.

\section{Bibliographie}

[1] Grosskreutz, J. C., Phys. Status Solidi (b) 47 (1971) 11.

[2] Grosskreutz, J. C., Int. Conf. Fracture (Munich) 1973, Vol. 1 PLV/212.

[3] Grosskreutz, J. C. et Mughrabi, H., Constitutive equations in Plasticity (Ed. A. S. Argon, MIT Press. Cambridge Mass.) 1975, p. 251.

[4] BAusChInger, J., Mitthlg des Mechanish-Technischen Lab. In Munchen, Heft XIII (1881) XXV.

[5] Ewing, J. A. et Humphrey, J. C. W., Philos. Trans. $R$. Soc. 200 (1903) 241.

[6] Gough, H. J., The Fatigue of Metals (E. Bruin Ltd. Londres) 1926.

[7] Cazaud, R., La Fatigue des Métaux (Dunod Paris) 1926.

[8] Coffin, L. F., Internal stresses and fatigue in Metals (Elsevier Publishing, New-York) 1959.

[9] Lieurade, H. P., La pratique des essais de fatigue (Société Française de Métallurgie, Paris) 1982.

[10] De Fouquet, J., Mughrabi, H., Bathias, C. et Pineau, A., Dislocations et déformation plastique (Ed. de Physique, Orsay) 1979, p. 355-393.

[11] Coulomb, P., Les textures dans les métaux de réseau cubique (Dunod, Paris) 1972.

[12] Coulomb, P., J. Micr. Spectr. Electr. 3 (1978) 295.

[13] Martin, J. L., J. Micr. Spectr. Electron. 1 (1976) 609.

[14] Veyssière, P., Dislocations et déformation plastique (Ed. de Physique, Orsay) 1979, p. 149.
[15] Strudel, J. L., J. Micr. Spectr. Electron. 3 (1978) 337.

[16] Thomas, B., J. Micr. Spectr. Electron. 1 (1976) 623.

[17] Vanderschaeve, G. et Escaig, B., Dislocations et déformation plastique (Ed. de Physique, Orsay) 1979 , p. 149.

[18] Christian, J. W., Proc. I.C.S.M.A. 3, Asilomar (A.S.M., Metal Park, Ohio) 1970, p. 31.

[19] Šesták, B. et Seeger, A., Z. Metallkunde 69 (1978) 195.

[20] Louchet, F., Kubin, L. P. et Veselý, D., Philos. Mag. 4 (1979) 443.

[21] Kubin, L. P., Rev. Def. Behaviour Mat. a) 1 (1976) 244, b) sous presse.

[22] Vitek, V., Cryst. Latt. Defects. 5 (1974) 1.

[23] Dotsenko, V. I., Phys. Status Solidi (b) 93 (1979) 11.

[24] Anglada, M. et Guiu, F., Scripta Met. 13 (1979) 103.

[25] Magnin, T., Thèse d'Etat (Université de Grenoble) 1980.

[26] Cagnon, M. et Groh, P., Dislocations et déformation plastique (Ed. de Physique, Orsay) 1979, p. 53 et 67.

[27] Laird, C., Finey, J. M. et Kuhlmann-Wilsdorf, D., Mat. Sci. Eng. 50 (1981) 127.

[28] Basinski, Z. S., Korbel, A. S. et BASInSKi, S. J., Acta Metall. 28 (1980) 191.

[29] BoOKer, G. R., Modern Diffraction and Imaging techniques in Materials Science (Ed. S. Amelinckx et al., North-Holland, Amsterdam) 1970, p. 597. 
[30] JALANTI, T., Impact de la Microscopie Electronique sur les Sciences Expérimentales (Ed. Ph. Buffat. E.P.F.L., Lausanne) 1979, p. 33.

[31] Mughrabi, H. et Wang, R., Int. Symp. Defects and Fracture. Tuczno-Pologne 1980) (Ed. G. C. Sih et H. Zorski, Martinus Nijhoff Publ., La Haye) 1982 , p. 15 .

[32] Bazinski, Z. S. et Bazinski, S. J., Proc. ICSMA 6, Melbourne (Ed. R. C. Gifkins, Pergamon Press, Oxford), 1982, Vol. 2, p. 819.

[33] Edington, J. W., Practical Electron Microscopy in Materials Science (Mc Millan Philips technical library, Eindhoven) 1975,5 Vol.

[34] Basinski, S. J., Basinski, Z. S. et Howie, A., Philos. Mag. 13 (1969) 899.

[35] Antonopoulos, J. G. et Winter, A. T., Philos. Mag. 33 (1976) 87.

[36] Mughrabi, H., J. Micr. Spectr. Electron. 1 (1976) 571

[37] Kubin, L. P. et Martin, J. L., Proc. ICSMA 5, Aachen (Ed. P. Haasen, V. Gerold et G. Kostorz, Pergamon Press, Oxford) 1979, Vol. 3, p. 1639.

[38] Yамамото, A. et al., High Voltage Electron Microscopy 1977 (Ed. T. Imura et H. Hashimoto, Japan Soc. for Electron Microscopy, Tokyo) 1971. p. 135.

[39] Yамамото, A. et Imura, T., Electron Microscopy 1980 (Ed. P. Brederoo et J. Van Landuyt, Electron Microscopy foundation, Leyden) 1980, p. 368.

[40] Lepinoux, J., Thèse 3e Cycle (Université de Poitiers) 1983.

[41] Lewandowski, P., Thèse Dr.-Ing. (Université Paul Sabatier, Toulouse) 1983.

[42] Tabata, T., Fujita, H., Hiraoka, M. et Onishi, K., Philos. Mag. A 47 (1983) 841.

[43] Driver, J. et RieuX, P., Mat. Sci. Eng. (1984), sous presse.

[44] Mughrabi, H., Mat. Sci. Eng. 33 (1978) 207.

[45] Avery, D. H. et Backofen, W. A., Acta Metal. 11 (1963) 653.

[46] Abel, A., Wilhelm, M. et Gerold, V., Mat. Sci. Eng. 37 (1979) 187.

[47] Woods, P. J., Philos. Mag. 28 (1973) 155.

[48] Winter, A. T., Philos. Mag. 30 (1974) 719.

[49] Feltner, C. E. et Laird, C., Acta Metal. 15 (1967) 1621 et 1633

[50] Ramaswami B., LaU, T. W. et Poon, G., Proc. ICSMA 5, Aachen (Ed. P. Haasen, V. Gerold et G. Kostorz, Pergamon Press, Oxford) 1979, p. 1169.

[51] Bhat, S. P. et LaIRD, C., Fatigue Mechanisms (Ed. J. T. Fong, ASTM-STP 675) 1979, p. 592.

[52] Blochwitz, C. et Veit, U., Cryst. Res. Technol. 17 (1982) 529.

[53] Mecke, K., Blochwitz, C. et Kremling, U., Cryst. Res. Technol. 17 (1982) 1557.

[54] Mecke, K. et Blochwitz, C., Cryst. Res. Technol. 17 (1982) 743

[55] Finney, J. M. et Laird, C., Philos. Mag. 31 (1975) 339.

[56] Neumann, P., a) Acta Metall. 17 (1969) 1219 et b) Constitutive equations in Plasticity (Ed. A. S Argon, MIT Press, Cambridge Mass. 1975, p. 449.

[57] Mughrabi, H., Proc. ICSMA 5, Aachen (Ed. P. Haasen, V. Gerold et G. Kostorz., Pergamon Press, Oxford) 1979, p. 1615
[58] Kuhlmann-Wilsdorf, D. et Laird, C., Mat. Sci. Eng. 27 (1977) 137.

[59] Winter, A. T., Philos. Mag. 37 (1978) 457.

[60] Brown, L. M., Proc. Int. Conf. on Dislocation Modelling of Physical Systems (Gainesville, Fla. Acta/Scripta Met.) 1980, p. 79.

[61] KuhlmanN-Wilsdorf, D. et Laird, C., Mat. Sci. Eng. 46 (1980) 209.

[62] Pedersen, O. B. et Winter, A. T., Acta Metall. 30 (1982) 711.

[63] Kuhlmann-Wilsdorf, D. et Laird, C., Mat. Sci. Eng 37 (1979) 111.

[64] Kuhlmann-Wilsdorf, D. et Laird, C., Proc. ICSMA 5, Aachen (Ed. P. Haasen, V. Gerold et G. Kostorz, Pergamon Press, Oxford) 1979, p. 1081.

[65] Mughrabi, H., Continuum Models of Discrete Systems 4 (Ed. O. Brulin et R. K. T. Hsieh, North-Holland) 1981, p. 241.

[66] Mughrabi, H., Ackermann, F. et Herz, K., Fatigue Mechanisms (Ed. J. T. Fong, ASTM-STP 675) 1979, p. 69.

[67] Wilhelm, M., Everwin, P., Proc. ICSMA 5, Aachen (Ed. P. Haasen, V. Gerold et G. Kostorz, Pergamon Press, Oxford) 1979, p. 1089.

[68] Mughrabi, H. et Wutrich, C., Philos. Mag. 33 (1976) 963.

[69] Magnin, T. et Driver, J., Low cycle fatigue and life prediction (Ed. C. Amzallay et al., ASTMSTP 770) 1982, p. 212

[70] Anglada, M. et Guiu, F., Philos. Mag. A 44 (1981) 499 et 523.

[71] Polak, J., Klesnil, M. et LuKas, P., Mat. Sci. Eng. 15 (1974) 231.

[72] Mughrabi, H., Z. Metallk. 66 (1975) 719.

[73] Lukas, P. et Polak, J., Work-hardening in tension and fatigue (Ed. A. W. Thompson, AIMMEE, NewYork) 1977 , p. 177

[74] Mughrabi, H., Herz, K. et Ackermann, F., Proc. ICSMA 4, Nancy (Ed. Laboratoire de Physique des Solides ENSMIM-INPL, Nancy) 1976, p. 1244.

[75] Magnin, T. et Driver, J., Mat. Sci. Eng., 39 (1979) 175

[76] Magnin, T., Driver, J. et Rieu, J., Mem. Sci. Rev. Met. 75 (1978) 293.

[77] Koss, D. A. et WoJciK, C. J., Met. Trans. 7A (1976) 1243.

[78] Etemad, B. et Guiu, F., Scripta Met. 8 (1974) 931.

[79] Anglada, M. et Guiu, F., Proc. ICSMA 5, Aachen (Ed. P. Haasen, V. Gerold et G. Kostorz, Pergamon Press, Oxford) 1979, p. 1237.

[80] Nine, H. D., J. Appl. Phys. 44 (1973) 4375

[81] Neumann, R. Z., Metallk. 66 (1975) 25.

[82] Magnin, T. et Driver, J., I.C.F. 5, Advances in Fracture research (Ed. D. François, Pergamon Press, Oxford) 1980, p. 307.

[83] Herz, K., Thèse (Université de Stuttgart) 1976.

[84] Mughrabi, H., Int. J. Fract. 17 (1981) 193.

[85] ACKermann, F., Thèse (Université de Stuttgart) 1981.

[86] Mori, H., Tokuwame, M. et Miyazaki, T., Philos. Mag. A 40 (1979) 409.

[87] Mughrabi, H., Herz, K. et Stark, X., Acta Metall. 24 (1976) 659.

[88] Magnin, T., Fourdeux, A. et Driver, J. Phys. Status Solidi a 65 (1981) 301. 